\title{
Reproducible experiments on Three-Dimensional Entity Resolution with JedAI
}

\author{
George Mandilaras ${ }^{1}$, George Papadakis ${ }^{1 *}$, Luca Gagliardelli², Giovanni Simonini ${ }^{2}$, \\ Emmanouil Thanos ${ }^{3}$, George Giannakopoulos ${ }^{4}$, Sonia Bergamaschi ${ }^{2}$, Themis Palpanas, \\ Manolis Koubarakis $^{1}$, Alicia Lara-Clares ${ }^{6 * *}$, Antonio Fariña $^{7 * *}$ \\ ${ }^{1}$ National and Kapodistrian University of Athens, Greece \{gmandi, gpapadis, koubarak\}@di.uoa.gr \\ ${ }^{2}$ University of Modena and Reggio Emilia, Italy \{name. surname\}@unimore. it \\ ${ }^{3} \mathrm{KU}$ Leuven, Belgium emmanouil.thanos@kuleuven.be \\ ${ }^{4}$ NCSR "Demokritos", Greece ggianna@iit.demokritos.gr \\ ${ }^{5}$ University of Paris $\mathcal{F}$ French University Institute (IUF), France themis@mi.parisdescartes.fr \\ ${ }^{6}$ NLPEIR Research Group, Universidad Nacional de Educación a Distancia (UNED), Spain alara@lsi.uned.es \\ ${ }^{7}$ University of A Coruña, CITIC, Database Lab, Spain antonio.farina@udc. es
}

\begin{abstract}
In Papadakis et al. [1], we presented the latest release of JedAl, an open-source Entity Resolution (ER) system that allows for building a large variety of end-to-end ER pipelines. Through a thorough experimental evaluation, we compared a schema-agnostic ER pipeline based on blocks with another schema-based ER pipeline based on similarity joins. We applied them to 10 established, real-world datasets and assessed them with respect to effectiveness and time efficiency. Special care was taken to juxtapose their scalability, too, using seven established, synthetic datasets. Moreover, we experimentally compared the effectiveness of the batch schema-agnostic ER pipeline with its progressive counterpart. In this companion paper, we describe how to reproduce the entire experimental study that pertains to JedAl's serial execution through its intuitive user interface. We also explain how to examine the robustness of the parameter configurations we have selected.
\end{abstract}

Keywords: Entity Resolution, Batch Methods, Progressive Methods, Reproducibility

\section{Introduction}

Entity Resolution (ER) is the task of identifying matches or duplicates, i.e., different entity profiles that describe the ${ }_{24}$ same real-world object. For example, ER should match the 25 entity profiles https://wwW.wikidata.org/wiki/Q30 26 and https://en.wikipedia.org/wiki/United_States, 27 which refer to the United States of America in two different ${ }_{28}$ data sources, Wikidate ${ }^{1}$ and Wikipedia ${ }^{2}$ respectively. ER constitutes a core data integration task and, thus, numerous ${ }_{29}$ approaches for tackling it have been proposed in the literature. ${ }_{30}$ Overviews of the main methods can be found in recent books ${ }_{31}$ [2, 3, 4, 5], surveys [6, 7, 8] and tutorials [9, 10, 11, 12].

To facilitate the use of the main ER methods, we created ${ }_{33}$ JedAI [1], an open-source system that allows for building end- ${ }_{34}$ to-end pipelines. JedAI enables users to effectively address the ER problem by categorizing the main methods into three orthogonal dimensions:

1. Schema-awareness categorizes ER methods into schemabased and schema-agnostic ones, depending on whether ${ }_{38}$ they rely on schema knowledge or not.

\footnotetext{
${ }^{*}$ Corresponding author

** Reviewer

1 https://www.wikidata.org

2 https://www.wikipedia.org
}

Preprint submitted to Elsevier
2. Budget-awareness categorizes ER methods into budgetagnostic ones, which operate as batch processes, and budget-aware ones, which operate in a pay-as-you-go manner that produces results progressively - they maximize the detected matches within a specific budget of temporal or computational resources.

3. Execution mode categorizes ER methods into serial and massively parallelized ones, e.g., over Apache Spark ${ }^{3}$

Using JedAl, we experimentally evaluated in [1] the relative performance of the main end-to-end ER pipelines that are defined by the three aforementioned dimensions. In this work, we focus on serially executed pipelines of any type.

Regarding schema-awareness, the schema-agnostic pipeline consists of the following steps, as shown in Figure 1.a):

- Data Reading loads the data to be processed into main memory.

- Schema Clustering is an optional step that groups together different attributes that share syntactically similar values so as to improve the performance of the subsequent steps. Note that this task differs from Schema Matching, which tries to identify the semantically matching attributes.

3 https://spark.apache.org 


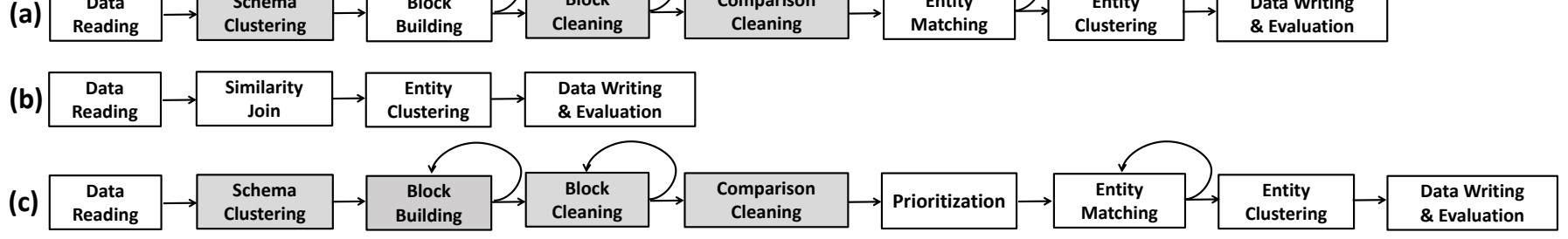

Figure 1: The three main end-to-end ER pipelines implemented by JedAl: (a) the budget- \& schema-agnostic one, (b) the budget-agnostic, schema-based one, and (c) the budget-aware, schema-agnostic one. Shaded rectangles indicate optional steps.

- Block Building aims to reduce the computational cost of 82 the brute-force approach, by limiting the search space to 8 similar entity profiles. To this end, it clusters together en- 84 tity profiles that share identical or similar signatures. $\quad 85$

- Block Cleaning is an optional step that further curtails the ${ }_{87}^{86}$ computational cost of ER by refining the output of Block ${ }_{88}$ Building. Its goal is actually to discard those blocks that are dominated by redundant and superfluous comparisons; the former involve pairs of entities co-occurring in multiple blocks, while the latter compare pairs of entities that 90 do not match.

- Comparison Cleaning is another optional step that serves ${ }_{92}$ the same purpose as Block Cleaning. It offers a more time- ${ }_{9}$ consuming, but more precise functionality that operates at the level of individual comparisons.

- Entity Matching estimates the matching likelihood for all entity pairs in the final set of blocks, using string similarity ${ }_{97}$ measures.

- Entity Clustering models the estimated similarities as a ${ }^{99}$ weighted, undirected graph and then partitions it into 100 equivalence clusters, i.e., disjoint sets of entity profiles ${ }_{101}$ that are considered as matches.

- Data Writing E Evaluation allows for storing the final re-10 sults and for assessing the performance of the selected ER pipeline with respect to the main effectiveness and time ${ }^{104}$ efficiency measures.

The schema-based end-to-end pipeline also starts with ${ }^{106}$ Data Reading and ends with Entity Clustering and Data Writing ${ }_{107}$ \& Evaluation, as shown in Figure 1(b). In between, it applies $\mathrm{a}_{108}$ single step, called Similarity Join, which rapidly estimates the ${ }_{109}$ pairs of entity profiles that satisfy a given matching rule, which $_{110}$ consists of:

1. a similarity measure,

2. the attribute on which the measure is applied, and

3. a threshold designating the minimum acceptable similarity ${ }^{114}$ for two entity profiles that are considered as matching.

As an example, consider the following matching rule for bibli-117 ographic entities: JaccardS im $\left(\right.$ title $_{1}$, title $\left._{2}\right)>0.8$.

In [1], we also compare the batch, schema-agnostic pipeline ${ }_{119}$ with its progressive counterpart, i.e., the budget-aware, 120 schema-agnostic pipeline, which is shown in Figure 11 (c). The only difference from the batch pipeline is the Prioritization step, which intervenes between Comparison Cleaning and Entity Matching. Its goal is to define the optimal processing order of the entity pairs in the final set of blocks so that the matching ones are detected as early as possible.

A video demonstrating JedAl in action is available at: https://www youtube . com/watch?v=0JY1DUrUAe8

\section{The reproducible experiments on Entity Resolution}

\subsection{Preliminaries}

Depending on the input data, Entity Resolution is categorized into two main categories:

1. Clean-Clean ER receives as input two datasets, which are individually duplicate-free (e.g., Wikipedia and Wikidata), and its goal is to identify the matches they share.

2. Dirty $E R$ receives as input one or more datasets, with at least one of them containing duplicates in itself. Its goal is to partition all entity profiles into equivalence clusters.

In both cases, the end-result of any end-to-end pipeline is evaluated with respect to three effectiveness measures:

- Recall assesses the portion of existing duplicates that are actually identified as such.

- Precision estimates the portion of entity pairs that are marked as matches and are indeed duplicates.

- F-Measure is the harmonic mean of Recall and Precision.

The progressive pipelines are additionally assessed through Progressive Recall, which quantifies the evolution of recall as more entity pairs are compared. We actually consider the area under its curve (AUC), which is derived from a twodimensional diagram, where horizontal axis corresponds to the number of executed comparisons and the vertical one to the number of detected duplicates. The larger (the area under the curve of) Progressive Recall is, the earlier are the matches identified and the better is the progressive pipeline.

All effectiveness measures are defined in the interval $[0,1]$, with higher values corresponding to higher effectiveness.

The time efficiency of an end-to-end pipeline is measured through its run-time, i.e., the time that intervenes between receiving the input entity profiles and producing the end result. 
Table 1: Technical characteristics of the Dirty ER datasets. $|E|$ stands for the number of entity profiles, NVP for the total number of name-value pairs in the dataset, $|N|$ for the number of distinct attributes, $|\bar{p}|$ for the average profile size (in terms of name-value pairs), $|D(E)|$ for the number of duplicate pairs, and $\|E\|$ for the comparisons executed by the brute-force approach.

\begin{tabular}{|l|rr||rrrrrrr|}
\cline { 2 - 10 } & \multicolumn{1}{c|}{$\mathbf{D}_{\text {cora }}$} & \multicolumn{1}{c|}{$\mathbf{D}_{\text {cddb }}$} & \multicolumn{1}{c|}{$\mathbf{D}_{\mathbf{1 0 K}}$} & \multicolumn{1}{c}{$\mathbf{D}_{\mathbf{5 0 K}}$} & \multicolumn{1}{c}{$\mathbf{D}_{\mathbf{1 0 0 K}}$} & \multicolumn{1}{c}{$\mathbf{D}_{\mathbf{2 0 0 K}}$} & \multicolumn{1}{c}{$\mathbf{D}_{\mathbf{3 0 0 K}}$} & \multicolumn{1}{c}{$\mathbf{D}_{\mathbf{1 M}}$} & $\mathbf{D}_{\mathbf{2 M}}$ \\
\hline \hline$|E|$ & 1,295 & 9,763 & 10,000 & 50,000 & 100,000 & 200,000 & 300,000 & $1,000,000$ & $2,000,000$ \\
$\mathrm{NVP}$ & 7,166 & 183,072 & 106,108 & 530,854 & $1,061,421$ & $2,123,728$ & $3,184,885$ & $10,617,729$ & $21,238,252$ \\
$|N|$ & 12 & 106 & 12 & 12 & 12 & 12 & 12 & 12 & 12 \\
$|\bar{p}|$ & 5.53 & 18.75 & 10.61 & 10.62 & 10.61 & 10.62 & 10.62 & 10.62 & 10.62 \\
$|D(E)|$ & 17,184 & 299 & 8,705 & 43,071 & 85,497 & 172,403 & 257,034 & 857,538 & $1,716,102$ \\
$\|E\|$ & $8.38 \cdot 10^{5}$ & $4.77 \cdot 10^{7}$ & $5.00 \cdot 10^{7}$ & $1.25 \cdot 10^{9}$ & $5.00 \cdot 10^{9}$ & $2.00 \cdot 10^{10}$ & $4.50 \cdot 10^{10}$ & $5.00 \cdot 10^{11}$ & $2.00 \cdot 10^{12}$ \\
\hline
\end{tabular}

Table 2: Technical characteristics of the Clean-Clean ER datasets.

\begin{tabular}{|l|r|r|r|r|r|r|r|r|}
\cline { 2 - 9 } \multicolumn{1}{c|}{} & \multicolumn{1}{c|}{$\mathbf{D}_{\mathbf{c} 1}$} & \multicolumn{1}{c|}{$\mathbf{D}_{\mathbf{c} 2}$} & \multicolumn{1}{c|}{$\mathbf{D}_{\mathbf{c} 3}$} & \multicolumn{1}{c|}{$\mathbf{D}_{\mathbf{c} 4}$} & \multicolumn{1}{c|}{$\mathbf{D}_{\mathbf{c} 5}$} & \multicolumn{1}{c|}{$\mathbf{D}_{\mathbf{c}}$} & $\mathbf{D}_{\mathbf{c} 7}$ & $\mathbf{D}_{\mathbf{c} 8}$ \\
\hline \hline Dataset $_{1}$ & Rest.1 & Abt & Amazon & DBLP & Walmart & DBLP & DBPedia & DBPedia 3.0rc \\
Dataset $_{2}$ & Rest.2 & Buy & Google Pr. & ACM & Amazon & Scholar & IMDB & DBPedia 3.4 \\
$\left|E_{1}\right| /\left|E_{2}\right|$ & $339 / 2,256$ & $1,076 / 1,076$ & $1,354 / 3,039$ & $2,616 / 2,294$ & $2,554 / 22,074$ & $2,516 / 61,353$ & $27,615 / 23,182$ & $1.19 \cdot 10^{6} / 2.16 \cdot 10^{6}$ \\
$\mathrm{NVP}_{1} / \mathrm{NVP}_{2}$ & $1,130 / 7,519$ & $2,568 / 2,308$ & $5,302 / 9,110$ & $10,464 / 9,162$ & $14,143 / 1.1 \cdot 10^{5}$ & $10,064 / 2 \cdot 10^{5}$ & $1.6 \cdot 10^{5} / 8.2 \cdot 10^{5}$ & $1.69 \cdot 10^{7} / 3.50 \cdot 10^{7}$ \\
$\left|N_{1}\right| /\left|N_{2}\right|$ & $7 / 7$ & $3 / 3$ & $4 / 4$ & $4 / 4$ & $6 / 6$ & $4 / 4$ & $4 / 7$ & $30,688 / 52,489$ \\
$\left|\bar{p}_{1}\right| /\left|\bar{p}_{2}\right|$ & $3.33 / 3.33$ & $2.39 / 2.14$ & $3.92 / 3.00$ & $3.99 / 4.00$ & $5.54 / 5.18$ & $3.23 / 3.26$ & $5.63 / 35.20$ & $14.19 / 16.18$ \\
$\left|D\left(E_{1} \cap E_{2}\right)\right|$ & 89 & 1,076 & 1,104 & 2,224 & 853 & 2,308 & 22,863 & 892,579 \\
$\left\|E_{1} \times E_{2}\right\|$ & $7.65 \cdot 10^{5}$ & $1.16 \cdot 10^{6}$ & $4.11 \cdot 10^{6}$ & $6.00 \cdot 10^{6}$ & $5.64 \cdot 10^{7}$ & $1.54 \cdot 10^{8}$ & $6.40 \cdot 10^{8}$ & $2.58 \cdot 10^{12}$ \\
\hline
\end{tabular}

Note that we also provide the minimum amount of main $_{150}$ memory that is required to successfully run each test in a way ${ }_{151}$ that approximates the lowest possible running time by minimiz-152 ing the impact of the garbage collector. The reported values cor-153 respond to the $-X m x$ parameter when running each experiment ${ }_{154}$ as a Java process, independently of Docker and the browser, 155 which raise additional memory requirements.

\subsection{Sets of Experiments}

The experimental analysis of [1] used 17 datasets. Each of ${ }_{159}$ them consists of one or two sets of entity profiles, in the case ${ }_{160}$ of Dirty and Clean-Clean ER, respectively, as well as a golden ${ }_{161}$ standard, i.e., the complete ground-truth of the actual duplicate entity profiles. They are all publicly available in the form of ${ }_{162}$ Java serialized objects as a Mendeley dataset [13] and through ${ }_{163}$ JedAl's repository ${ }^{4}$ Their technical characteristics are reported ${ }_{164}$ in Tables 1 and 2, which are the same as Tables 1 and 2 in [11],165 but are repeated here for convenience. Additional information ${ }_{166}$ about all datasets is provided in Table 3 .

Our experiments are divided into three sets as follows:

1. The Performance Tests examine the relative performance ${ }^{169}$ of the two budget-agnostic pipelines - the schema-based ${ }^{170}$ and the schema-agnostic one.

2. The Scalability Tests examine how the performance of the ${ }^{171}$ two budget-agnostic pipelines evolves as the size of the ${ }^{172}$ input data increases.

3. The Budget-awareness Tests examine the relative perfor- ${ }^{174}$ mance of the two forms of the schema-agnostic pipeline: ${ }_{175}$ the budget-agnostic and the budget-aware.

Below, we describe every set of experiments in more detail. ${ }^{178}$

$\sqrt[4]{\text { https://github.com/scify/JedAIToolkit }}$
Performance Tests. These experiments, which are reported in Table 4 of [1], compare the schema- and budget-agnostic pipeline with its schema-based counterpart over 10 real-world datasets. Two of them pertain to Dirty ER $\left(D_{\text {cora }}\right.$ and $\left.D_{c d d b}\right)$ and the rest to Clean-Clean ER $\left(D_{c 1}-D_{c 8}\right)$. The goal of these experiments is to evaluate both the relative effectiveness and the relative time efficiency of these pipelines. For the schema-agnostic pipeline, we consider two configurations:

1. the best one, which uses the parameters that maximize the F-Measure per dataset, and

2. the default one, which uses the default parameters for each method in the pipeline, thus being the same for all datasets.

For the schema-based pipeline, we exclusively consider the best configuration per dataset, which maximizes F-Measure.

Note that these tests involve two baseline systems that have been developed by other research groups, Magellan [26] and DeepMatcher [27]. Due to their human-in-the-loop approach and the lack of necessary details, we could not test their performance ourselves. Instead, we reported their top F-measure per dataset in [27], among all configurations and dataset versions. For this reason, we disregard both systems in the following.

Scalability Tests. These experiments are described in the diagrams of Figure 7 in [1], comparing again the two budgetagnostic end-to-end pipelines. In this case, though, the goal is to assess how their time efficiency and effectiveness evolve as the size of the data increase from several thousand to few million entity profiles. To this end, we use seven datasets that pertain exclusively to Dirty ER; their names indicate their size, i.e., the number of their entity profiles: $D_{10 K}, D_{50 K}$, $D_{100 K}, D_{200 K}, D_{300 K}, D_{1 M}$ and $D_{2 M}$. These datasets contain synthetic census data, i.e., information about individuals that has been enriched with various forms of artificial 
Table 3: Core information about each dataset: its reference work, its type (i.e., whether it involves real or synthetic data), the corresponding ER task (Clean-Clean or Dirty ER), the paths of its entity profiles and its golden standard files in the data repository of [13] and the original data source. We have categorized the 17 datasets in three groups according to their type and task, following [13], which contains a different folder for each group. Note that in [13], all parts of $D_{c 8}$ are provided through a single zipped file, newDBPedia.tar. xz, to minimize their large size.

\begin{tabular}{|c|c|c|c|c|c|}
\hline Dataset & Type & Task & Path to the Entity Profiles File in [13] & Path to the Golden Standard File in [13] & Source \\
\hline $\mathbf{D}_{\mathbf{c 1}}$ & Real & Clean-Clean ER & $\begin{array}{l}\text { Real Clean-Clean ER data/restaurant1Profiles } \\
\text { Real Clean-Clean ER data/restaurant2Profiles }\end{array}$ & Real Clean-Clean ER data/restaurant1IdDuplicates & 15 \\
\hline $\mathbf{D}_{\mathbf{c 2}}$ & Real & Clean-Clean ER & $\begin{array}{l}\text { Real Clean-Clean ER data/abtProfiles } \\
\text { Real Clean-Clean ER data/buyProfiles }\end{array}$ & Real Clean-Clean ER data/abtBuyIdDuplicates & 17] \\
\hline $\mathbf{D}_{\mathbf{c 3}}$ & Real & Clean-Clean ER & $\begin{array}{r}\text { Real Clean-Clean ER data/amazonProfiles } \\
\text { Real Clean-Clean ER data/gpProfiles }\end{array}$ & Real Clean-Clean ER data/amazonGpIdDuplicates & 17 \\
\hline $\mathbf{D}_{\mathrm{c} 4} 16$ & Real & Clean-Clean ER & $\begin{array}{l}\text { Real Clean-Clean ER data/dblpProfiles } \\
\text { Real Clean-Clean ER data/acmProfiles }\end{array}$ & Real Clean-Clean ER data/dblpAcmProfiles & 17 \\
\hline $\mathbf{D}_{\mathrm{c5}}$ & Real & Clean-Clean ER & $\begin{array}{l}\text { Real Clean-Clean ER data/walmartProfiles } \\
\text { Real Clean-Clean ER data/amazonProfiles2 }\end{array}$ & Real Clean-Clean ER data/amazonWalmartIdDuplicates & 19 \\
\hline Dc6 16 & Real & Clean-Clean ER & $\begin{array}{r}\text { Real Clean-Clean ER data/dblpProfiles2 } \\
\text { Real Clean-Clean ER data/scholarProfiles }\end{array}$ & Clean-Clean ER data/dblpScholarIdDuplicates & 17 \\
\hline $\mathbf{D}_{\mathbf{c} 7}$ [20] & Real & Clean-Clean ER & $\begin{array}{r}\text { Real Clean-Clean ER data/imdbProfiles } \\
\text { Real Clean-Clean ER data/dbpediaProfiles }\end{array}$ & Clean-Clean ER data/moviesIdDuplicates & [21] \\
\hline $\mathbf{D}_{\mathbf{c 8}}$ 20] & Real & Clean-Clean ER & $\begin{array}{l}\text { Real Clean-Clean ER data/cleanDBPedia1 } \\
\text { Real Clean-Clean ER data/cleanDBPedia2 }\end{array}$ & Clean-Clean ER data/newDBPediaMatches & 21 \\
\hline $\begin{array}{l}\left.D_{\text {cora }} \mid 22\right] \\
D_{\text {cddb }} \mid 24\end{array}$ & $\begin{array}{l}\text { Real } \\
\text { Real }\end{array}$ & $\begin{array}{l}\text { Dirty ER } \\
\text { Dirty ER }\end{array}$ & $\begin{array}{l}\text { Real Dirty ER data/coraProfiles } \\
\text { Real Dirty ER data/cddbProfiles }\end{array}$ & $\begin{array}{l}\text { Real Dirty ER data/coraIdDuplicates } \\
\text { Real Dirty ER data/cddbIdDuplicates }\end{array}$ & 23 \\
\hline$\left.\overline{\mathbf{D}_{10 K}} \mid 25\right]$ & Synthetic & Dirty ER & Synthetic Dirty ER data/10Kprofiles & "Synthetic Dirty ER data/10KIdDuplicates & 21 \\
\hline $\mathbf{D}_{50 \mathrm{~K}} 25$ & Synthetic & Dirty ER & Synthetic Dirty ER data/50Kprofiles & Synthetic Dirty ER data/50KIdDuplicates & 21] \\
\hline$D_{100 K} 25$ & Synthetic & Dirty ER & Synthetic Dirty ER data/100Kprofiles & Synthetic Dirty ER data/100KIdDuplicates & 21 \\
\hline $\mathbf{D}_{200 \mathrm{~K}} 25$ & Synthetic & Dirty ER & Synthetic Dirty ER data/200Kprofiles & Synthetic Dirty ER data/200KIdDuplicates & 21] \\
\hline $\mathbf{D}_{300 \mathrm{~K}} 25$ & Synthetic & Dirty ER & Synthetic Dirty ER data/300Kprofiles & Synthetic Dirty ER data/300KIdDuplicates & 21 \\
\hline $\mathbf{D}_{1 \mathrm{M}} 25$ & Synthetic & Dirty ER & Synthetic Dirty ER data/1Mprofiles & Synthetic Dirty ER data/1MIdDuplicates & [21] \\
\hline $\mathbf{D}_{2 M}$ & Synthetic & Dirty ER & Synthetic Dirty ER data/2Mprofiles & Synthetic Dirty ER data/2MIdDuplicates & [21] \\
\hline
\end{tabular}

noise (see [1] for more details). For both pipelines, we con-206 sider a single configuration that is applied to all datasets:207 the default configuration for the schema-agnostic pipeline $\operatorname{and}_{208}$ the matching rule that consistently achieves reasonable per-209 formance across all datasets for the schema-based one, i.e.,210 JaccarSim(all_tokens_1,all_tokens_2) $>0.4$, executed by 211 PPJoin and followed by Connected Components with the same 212 similarity threshold.

Budget-awareness Tests. These experiments are reported in ${ }^{214}$ the diagrams of Figure 8 in [1]. They compare the budget- ${ }_{216}$ and schema-agnostic pipeline with its budget-aware counterpart ${ }_{217}$ across the same datasets as the Performance Tests - except the ${ }_{218}$ largest one, $D_{c 8}$. For each dataset, the parameter configuration ${ }_{219}$ that corresponds to the optimal performance of the budget- $\operatorname{and}_{220}$ schema-agnostic pipeline is also used for the common meth- ${ }_{221}$ ods of its budget-aware version. In this way, these tests assess the impact of the Prioritization step, which constitutes the sole $_{223}$ difference between the two pipelines. We evaluate the time effi- ${ }_{224}$ ciency of the two workflows through their running times and the ${ }_{225}$ effectiveness through the area under their Progressive Recall.

\subsection{Experimental setup in our primary paper}

All single-core experiments in [1] were implemented in Java 229 8 and can be reproduced through JedAl's Docker image, which is publicly available ${ }^{5}$ The only requirement is to have Docker ${ }^{6}$

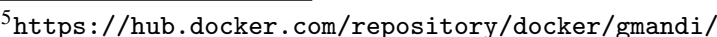
jedai-webapp

https://www.docker.com installed. Table 4 provides detailed instructions for installing the latest version of Docker on Ubuntu. A similar procedure is required for other Linux distributions, like Debian. ${ }^{7}$ Fedor ${ }^{8}$ and CentOS ${ }^{9}$ JedAl's Docker image is expected to run seamlessly in all these cases. Upon successful completion of these commands, JedAl's Web application appears in a browser at: http://localhost: 8080.

Note that the option -e JAVA_OPTIONS=' $-\mathrm{Xmx} 4 \mathrm{~g}$ ' determines that 4 Gigabytes (GB) of RAM memory is allocated to Java to run JedAl's Web application. This is an optional parameter, as the vast majority of our experiments can be run with much fewer memory, as indicated by the memory requirements that are reported in Tables 8,9 and 10 for each experiment. In our tests, though, we noticed that $4 \mathrm{~GB}$ are more suitable for ensuring Docker's stability. Otherwise, it needs restarting after some tests. When experimenting with larger datasets, it is actually recommended to devote all or most of the available memory to Docker so as to avoid out-of-memory exceptions or excessively large running times, due to the overuse of the garbage collector.

Note also that the option - $\mathrm{v} / \mathrm{absolute/path}$ is necessary because JedAl's Docker starts by downloading all datasets from the Mendeley data repository [13]. Thus, this option determines the directory on the host system (e.g., /home/user/jedai),

${ }^{7}$ See https://docs.docker.com/engine/install/debian for detailed instructions.

${ }^{8}$ See https://docs.docker.com/engine/install/fedora for detailed instructions.

${ }^{9}$ See https://docs.docker.com/engine/install/centos for detailed instructions. 
Table 4: Detailed instructions for installing and running JedAl's Docker image on Ubuntu. The steps 1-7 install the latest version of Docker Community Edition. For more details, please refer to the official Docker setup page at: https://docs.docker.com/engine/install/ubuntu The remaining steps download JedAl's Docker image from the Docker Hub (step 8) or from JedAl's Mendeley data repository (step 8') and execute it (step 9).

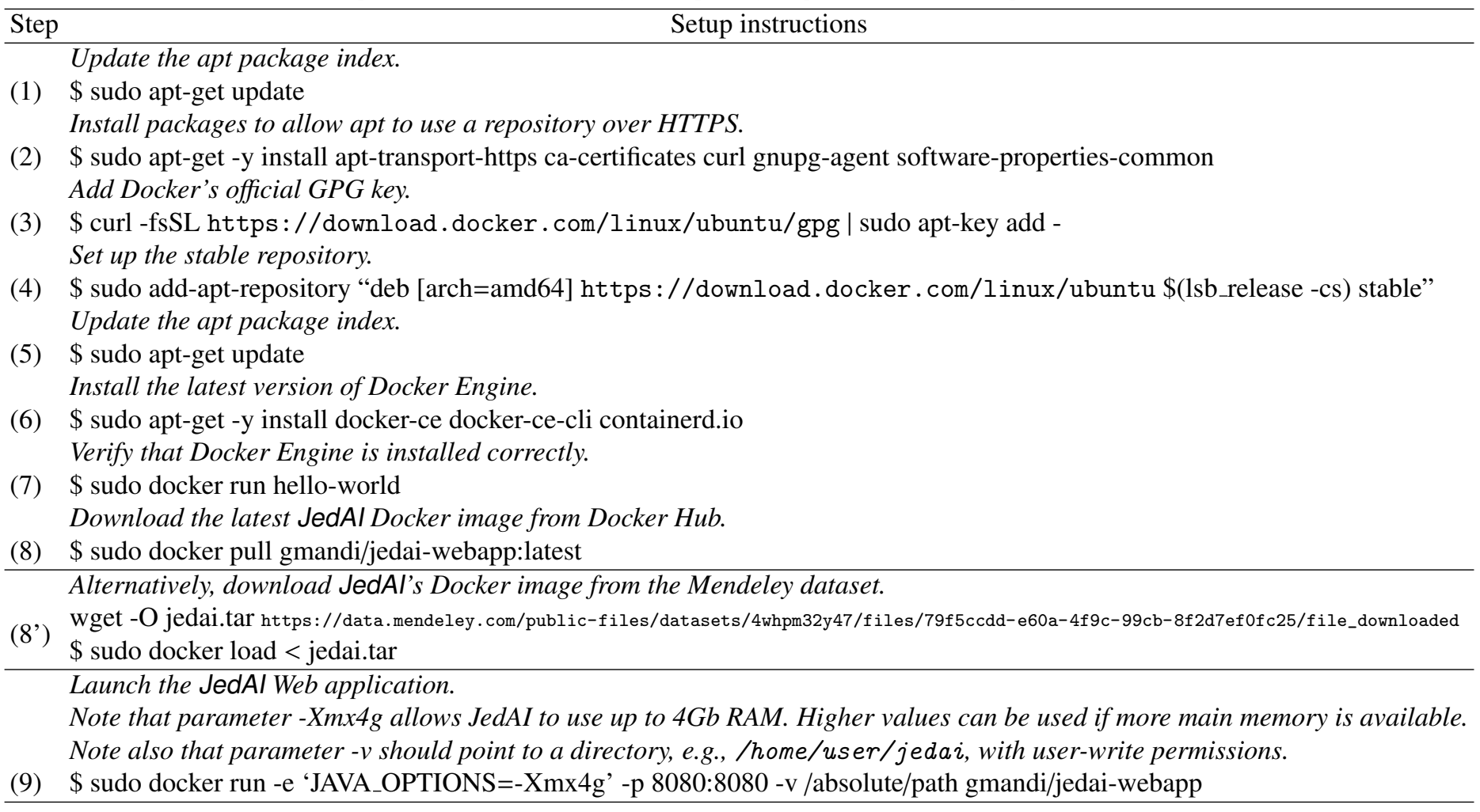

where Docker will store and unpack the dataset files as long as 252 it has user-write permissions.

It is also worth noting that in the option $-\mathrm{p}$ 8080:8080, the 254 first 8080 refers to the host port, and could be replaced by any 255 other free port in the host. Docker will map the first port $\underline{8080}_{256}$ to the http port (second 8080) from the docker container.

Finally, it is worth noting that it is also possible to use Docker 258 on Windows 10. The installation is a straightforward proce-259 dure ${ }^{10}$ that merely needs some additional steps ${ }^{11}$ After the 260 successful installation, all experiments can be seamlessly run,261 without any performance issue. Indeed, one of our testing plat-262 forms runs on Windows 10 Pro (Windows - base 1 in Table 5). 263

\subsection{System requirements and performance evaluation}

All single-core experiments in [1] can be reproduced on any ${ }^{266}$ Java 8 compliant platform, which practically includes all major ${ }^{267}$ Linux distributions. Our experiments have been successfully ${ }^{268}$ reproduced on all testing platforms reported in Table 5, with the ${ }^{269}$ aggregate running times that are reported in Table 6 . Note that ${ }^{270}$ in all systems, a single CPU core was used for each experiment. ${ }^{271}$

Our original configuration corresponds to Ubuntu-base 1 for $^{272}$ the Performance and Scalability Tests and to Ubuntu - base $1^{\prime 273}$ for the Budget-awareness Tests. Ubuntu - base 2 is a similar ${ }^{274}$

\footnotetext{
${ }^{10}$ See https ://docs .docker.com/docker-for-windows/install for detailed instructions.

${ }^{11}$ See https://docs.docker.com/docker-for-windows/wsl for more details.
}

server but with a different CPU that accounts for significant diversity in the running times. A more important difference is that in Ubuntu - base 1 and Ubuntu - base 1', all experiments were run through script files ${ }^{12}$ whereas in Ubuntu - base2, the experiments were carried out through the user interface of JedAl's Web application. The same applies to all other systems.

Among the other platforms, it is worth stressing that Ubuntu-base 4 consists of a bootable USB stick that runs a live Ubuntu instance on top of a Windows 10 laptop. The only implication was that it required a different approach for installing Docker ${ }^{13}$ No performance issue arose. In fact, Ubuntu-base 4 is often one of the fastest testing platforms, due to the newer generation of CPU and RAM technology.

Regarding the minimum system specifications required by our experiments, the size of the hard disk plays a minor role. Given that all experiments are executed in main memory and produce no output files, the hard disk requirements are determined by the space occupied by the Java JDK and the Docker installation as well as the size of JedAl's Docker image, which also includes all datasets. In total, this amounts to around 4 $\mathrm{GB}$, assuming an underlying blank Ubuntu installation. Note, though, that this space is occupied whenever command 9 in Table 4 is executed. To recover the space occupied after multiple

\footnotetext{
${ }^{12}$ The source code of all tests is available at: https://github.com/ scify/JedAIToolkit/tree/master/src/test/java/org/scify/ jedai/version3

${ }^{13}$ For more details, please refer to https://stackoverflow.com/ questions/30248794/run-docker-in-ubuntu-live-disk
} 
Table 5: The testing platforms that were successfully used to reproduce our experiments. Note that Ubuntu-base 1 was used in [1] for performing the experiments reported in Tables 8 and 9 while Ubuntu - base $1^{\prime}$ was only used for the experiments in Table 10

\begin{tabular}{|c|c|c|c|c|}
\hline Testing platform & Type & Software Configuration & Hardware Configuration & Tested by \\
\hline Ubuntu-base 1 & Server & $\begin{array}{l}\text { Ubuntu 14.04.5 LTS } \\
\text { OpenJDK 1.8.0 }\end{array}$ & $\begin{array}{l}\text { 1 Intel Xeon E5-4603 v2 @ 2.20GHz, } \\
128 \text { Gb DDR3 RAM, 1.6 Tb mechanical disk }\end{array}$ & Authors \\
\hline Ubuntu-base $1^{\prime}$ & Server & $\begin{array}{l}\text { Ubuntu 14.04 LTS } \\
\text { Java 1.8.0 }\end{array}$ & $\begin{array}{l}\text { 1 Intel Xeon E5-2670 v2 @ 2.50GHz, } \\
\text { 80GB DDR3 RAM, 1Tb mechanical disk }\end{array}$ & Authors \\
\hline Ubuntu-base 2 & Server & $\begin{array}{l}\text { Ubuntu 14.04.6 LTS } \\
\text { Docker 19.03.13, Java 1.8.0 }\end{array}$ & $\begin{array}{l}\text { 1 AMD Opteron } 6320 @ 2.80 \mathrm{GHz}, \\
128 \mathrm{~Gb} \text { DDR3 RAM, } 1.6 \mathrm{~Tb} \text { mechanical disk }\end{array}$ & Authors \\
\hline Ubuntu-base3 & Laptop & $\begin{array}{l}\text { Ubuntu 18.04.5 LTS } \\
\text { Docker } 20.10 .5, \text { Java } 1.8 .0\end{array}$ & $\begin{array}{l}1 \text { Intel Core i7-4710MQ @2.50GHz, } \\
16 \text { Gb DDR3 RAM, } 120 \text { Gb SSD }\end{array}$ & Authors \\
\hline Ubuntu-base4 & Laptop & $\begin{array}{l}\text { Ubuntu 20.04 LTS } \\
\text { Docker 19.03.8, OpenJDK } 1.8 .0\end{array}$ & $\begin{array}{l}1 \text { Intel Core i5-1035G1 @ 1.00GHz, } \\
4 \text { Gb DDR4 RAM, } 32 \text { Gb flash drive }\end{array}$ & Authors \\
\hline Ubuntu-base 5 & Laptop & $\begin{array}{l}\text { Linux Mint 19.1 Tessa } \\
\text { Docker 19.03.8, Java } 1.8 .0\end{array}$ & $\begin{array}{l}1 \text { Intel Core i7-3770 @ 3.40GHz, } \\
16 \text { Gb DDR3 RAM, } 1 \text { Tb mechanical disk }\end{array}$ & Authors \\
\hline Ubuntu-base6 & Laptop & $\begin{array}{l}\text { Ubuntu 20.04.2 LTS } \\
\text { Docker 19.03.14, OpenJDK 1.8.0 }\end{array}$ & $\begin{array}{l}\text { Intel Core i7-9750H @ } 2.60 \mathrm{GHz} \\
32 \text { GB RAM, } 2.5 \mathrm{~Tb} \text { mechanical disk }\end{array}$ & Reviewer \\
\hline Ubuntu-base7 & Server & Ubuntu 20.04.2 LTS & $\begin{array}{l}\text { 1 Intel Xeon Bronze } 3204 \text { @ 1.9GHz, } \\
512 \text { Gb DDR4 RAM,120Gb mechanical disk }\end{array}$ & Reviewer \\
\hline Ubuntu-base8 & Server & Ubuntu 16.04.7 LTS & $\begin{array}{l}1 \text { Intel Core i7 8700k @3.7GHz, 64Gb swap, } \\
64 \text { Gb DDR4 RAM, 3Tb mechanical disk }\end{array}$ & Reviewer \\
\hline Ubuntu-base 9 & Laptop & Ubuntu 20.04.1 LTS & $\begin{array}{l}1 \text { Intel Core i5 8265u @ 1.6GHz, 16 DDR4 RAM, } \\
\text { no swap, 34Gb virtual disk over SSD }\end{array}$ & Reviewer \\
\hline Windows - base 1 & Laptop & $\begin{array}{l}\text { Windows } 10 \text { Pro v. } 20 \mathrm{H} 2, \\
\text { Docker } 20.10 .5, \text { Java } 15.0 .1\end{array}$ & $\begin{array}{l}1 \text { Intel Core i5-1035G1 @ 1.00GHz, } \\
6 \text { Gb DDR4 RAM, } 240 \text { Gb SSD }\end{array}$ & Authors \\
\hline
\end{tabular}

Table 6: The aggregate time required to run all the experiments included in ${ }^{283}$ Tables 8 and 10 (that could be completed in less than 40 hours) for each $^{284}$ testing platform, while reproducing most experiments from [1]. The testing platforms Ubuntu - base3, Ubuntu - base4, Ubuntu-base5, Ubuntu-base6, Ubuntu - base 9 and Windows - base 1 were limited in some experiments by the available main memory, thus exhibiting lower aggregate running times. $\quad 285$

\begin{tabular}{|c|c|c|c|}
\hline Run & Testing platform & Running time & Tested by \\
\hline 1 & Ubuntu-base 1 & $5,526 \mathrm{~min} \approx 92.1 \mathrm{hrs}$ & Authors \\
\hline 2 & Ubuntu-base2 & $6,832 \mathrm{~min} \approx 113.9 \mathrm{hrs}$ & Authors \\
\hline 3 & Ubuntu-base3 & $2,678 \mathrm{~min} \approx 44.6 \mathrm{hrs}$ & Authors \\
\hline 4 & Ubuntu-base4 & $187 \mathrm{~min} \approx 3.1 \mathrm{hrs}$ & Authors \\
\hline 5 & Ubuntu-base5 & $2,198 \mathrm{~min} \approx 36.6 \mathrm{hrs}$ & Authors \\
\hline 6 & Ubuntu-base6 & $1,428 \mathrm{~min} \approx 23.8 \mathrm{hrs}$ & Reviewer \\
\hline 7 & Ubuntu-base7 & $6,393 \mathrm{~min} \approx 106.5 \mathrm{hrs}$ & Reviewer \\
\hline 8 & Ubuntu-base8 & $3,212 \mathrm{~min} \approx 53.5 \mathrm{hrs}$ & Reviewer \\
\hline 9 & Ubuntu - base 9 & $1,731 \mathrm{~min} \approx 28.8 \mathrm{hrs}$ & Reviewer \\
\hline 10 & Windows - base 1 & $1,743 \mathrm{~min} \approx 29.1 \mathrm{hrs}$ & Authors \\
\hline
\end{tabular}

\section{runs, we can:}

- Remove the existing Docker containers: sudo docker container ls -a I grep gmandi obtains the IDs of JedAl's containers, and sudo docker $r m-f$ containerID removes a given container.

- Remove JedAl's Docker image: sudo docker rmi gmandi/jedai-webapp ${ }^{14}$

\footnotetext{
${ }^{14}$ Alternatively, run sudo docker images to obtain the IDs of the images, ${ }^{308}$ and then use sudo docker rmi imageID to remove them.
}

- Finally, recover disk space for unused volumes: sudo docker volume prune.

Regarding the size of main memory (RAM), the vast majority of experiments require less than $2 \mathrm{~Gb}$, as reported in Tables 8. 9 and 10 but $4 \mathrm{~Gb}$ are suggested to ensure Docker's stability, as explained above. However, the experiments with the two largest synthetic datasets, $D_{1 M}$ and $D_{2 M}$, require up to 25 $\mathrm{Gb}$, whereas the largest real dataset, $D_{c 8}$, requires up to 105 $\mathrm{Gb}$. The corresponding experiments cannot be run on most testing platforms that are equipped with $16 \mathrm{~Gb}$ RAM or less, namely Ubuntu - base3, Ubuntu - base4, Ubuntu - base5, Ubuntu - base6, Ubuntu - base 9 and Windows - base1. Below, we report in detail the memory requirements of every experiment, highlighting the experiments that were not feasible, due to insufficient main memory in the testing platforms.

Finally, it is worth noting that the times reported in Table 6 merely correspond to the time taken by each system to run all experiments. Given that each experiment is carried out through the user interface of JedAl's Web application (i.e., they are not executed through a script), significant time is taken to manually navigate through all menus. Among them, the Entity Matching step requires additional time to transform the selected dataset into the textual representation that is suitable for assessing entity similarity (e.g., by tokenizing all attribute values into character n-grams). This time, which is negligible only for the smallest datasets, is not added to the overall running times in Table 6, which disregard completely the navigation time. 
Table 7: Detailed instructions for reproducing all single-core experiments in [1] using the graphical user interface of JedAl's Web application.

Step Reproduction instructions

After launching JedAl's Docker image with the last command in Table 4

(1) Open a browser at http://localhost:8080.

If Docker runs on a server, replace 'localhost with its URL. The host port 8080 was arbitrarily specified by the last command in Table 4 and can be changed at will. JedAl's homepage, depicted in Figure 2 (a), shows up.

(2) Press the button 'New Workflow'.

The window 'Choose New Workflow mode' in Figure 2 b) pops up.

(3) Press the button 'Desktop Mode'.

Because we are interested in the serial execution of JedAl's experiments.

The Web page 'Select Workflow' in Figure 2 (c) shows up.

(4) Press the button 'Run tests' at the bottom right corner.

The window 'Select Test to execute' in Figure 22 (d) shows up.

The web application is already equipped with the parameters of all experiments.

Thus, any experiment in [1] can be reproduced simply by selecting it from the menus of Figure 2 (d).

(5) In 'Test Type', select 'Performance Test', 'Scalability Test' or 'Budget-awareness Test'.

The options for the rest of the selection criteria in the same window are activated.

(6) In 'ER Mode', select 'Clean-Clean ER' or 'Dirty ER'.

For Scalability Tests, only 'Dirty ER' is available.

(7) In 'Workflow Type', select 'Best Schema-agnostic', 'Default Schema-agnostic' or 'Schema-aware' pipelines. For Scalability Tests, only the last two options are available.

(8) In 'Datasets', select one among the available datasets in Tables 1, 2 and 3

(9) Press the button 'Confirm'.

JedAl loads the selected pipeline with the parameter configuration corresponding to the selected dataset.

One Web page for each step in the selected pipeline (see Figure 1) shows up.

(10) Press the button 'Next' in the window of each pipeline step to proceed to the next one.

After going through all pipeline steps, the Web page 'Confirm Configurations' in Figure 2 (e) shows up.

(11) Press the button 'Confirm'.

The Web page 'Workflow Execution' shows up.

(12) Press the button 'Execute Workflow'.

The selected experiment is carried out. Upon completion, the respective performance is reported in the same window with respect to Recall, Precision, F-Measure and running time, as in Figure $2(f)$.

(13) In case of Budget-awareness Tests, press the button 'Show Plot' at the bottom left corner.

A window similar to the one in Figure 2 (g) shows up, depicting Progressive Recall along with the area under its curve.

(14) Press JedAl logo at the top of the window to return to the first screen and proceed with the next test.

\subsection{Obtaining and compiling our source code}

The source code for JedAl version 3.0, which is used $\mathrm{in}^{32}$ [1] and in the present experimental study, has been publicly ${ }^{330}$ released at: https://github.com/scify/JedAIToolkit Any development kit and/or IDE for Java 8 or higher can be used for compiling it, but this is not necessary. JedAl's Docker ${ }^{333}$ image contains an executable jar file with the entire source code ${ }^{334}$ and its dependencies. When executed, it deploys JedAl's Web ${ }^{335}$ application, allowing users to reproduce all experiments by fol- ${ }^{336}$ lowing the instructions below, in Section 2.6

\subsection{Running the experiments}

Table 7 provides detailed guidelines for reproducing all ex-341 periments. In essence, the user merely needs to navigate ${ }_{342}$ through the windows of JedAl's user interface, which are il-343 lustrated in Figure 2. This means that minimal human interven-344 tion is required. For example, all datasets in Tables 1,2 and 3,45 are already included in JedAl's Docker image; the one selected ${ }_{346}$ in Step 8 is automatically loaded after the Data Reading step, 347 ara used in the chosen experiment is already marked as selected and
its parameters are appropriately configured. The user simply needs to press 'Next' in each step to proceed with the next one.

It is worth stressing at this point the wealth of information that is provided by the final window, called 'Workflow Execution', after completing an experiment:

1. The button 'Explore' presents the entity profiles that form each equivalence cluster.

2. The tab 'Details' contains the output of each step in the latest pipeline so as understand its operation and contribution to the overall performance.

3. The tab 'Workbench' summarizes the performance of all pipelines executed so far, as shown in Figure 2(h). This allows for juxtaposing the performance of different pipelines over the same dataset, even at the level of individual steps: pressing the button $\equiv$ in the leftmost column displays a performance breakdown among all steps. 


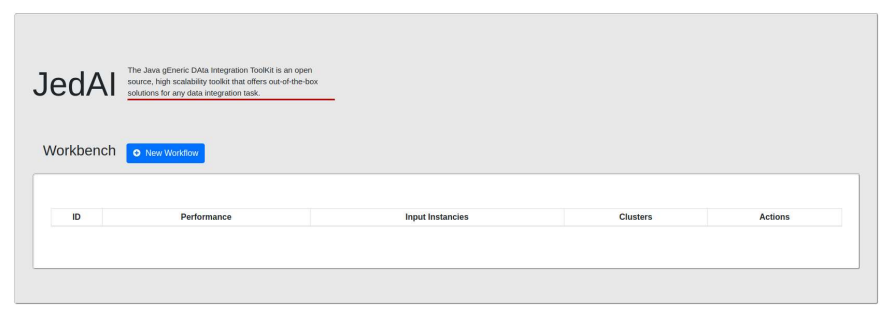

(a)

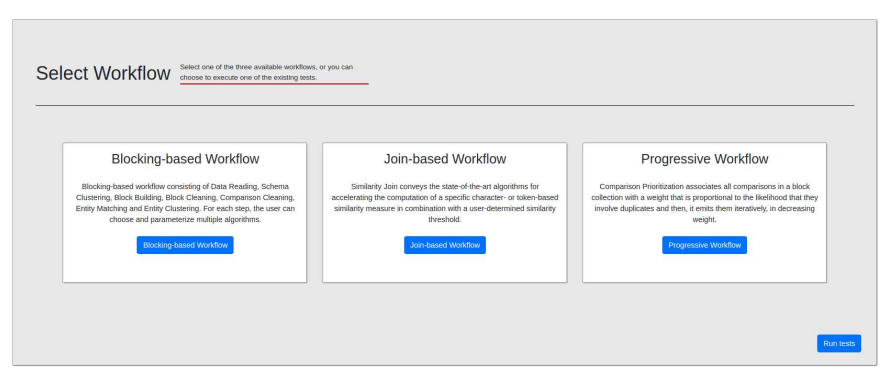

(c)

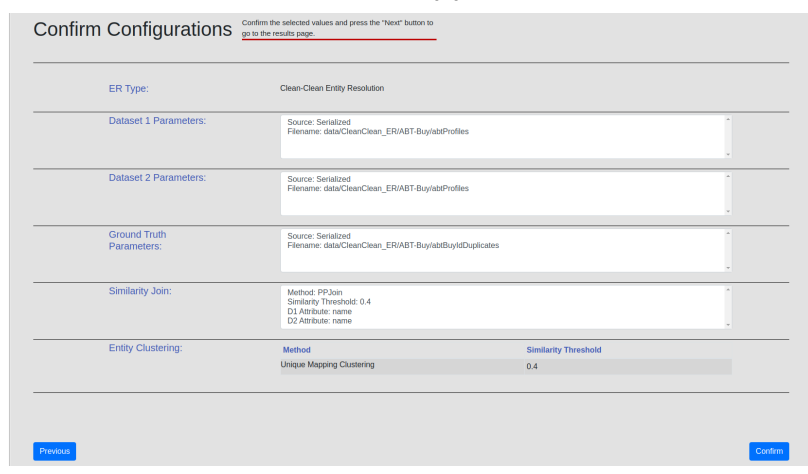

(e)

ROC Curve

AUC: 0.709

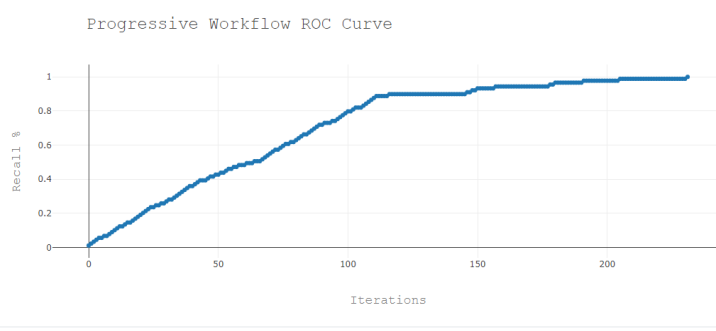

(g)

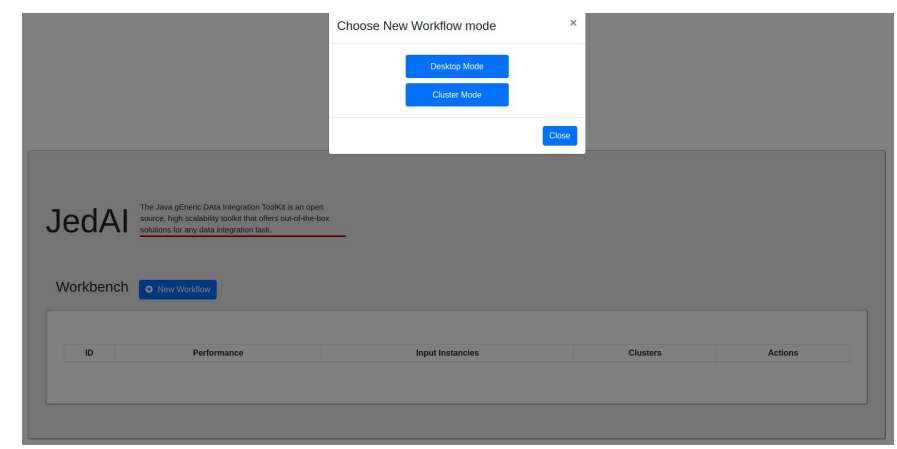

(b)

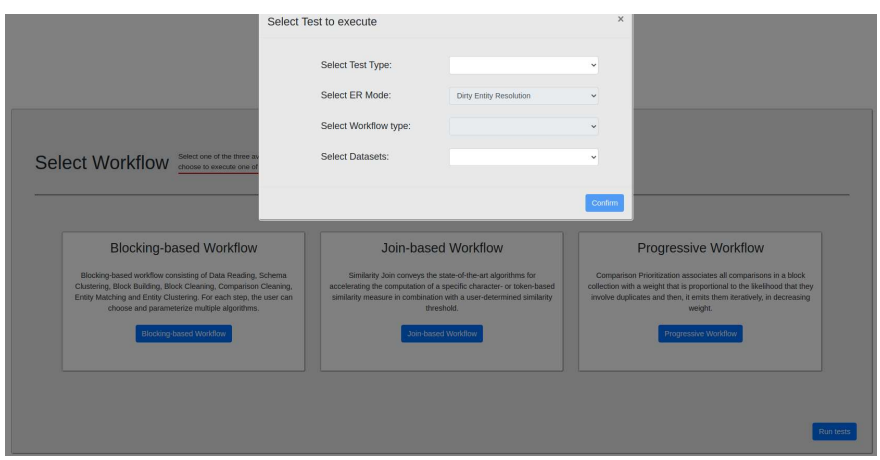

(d)

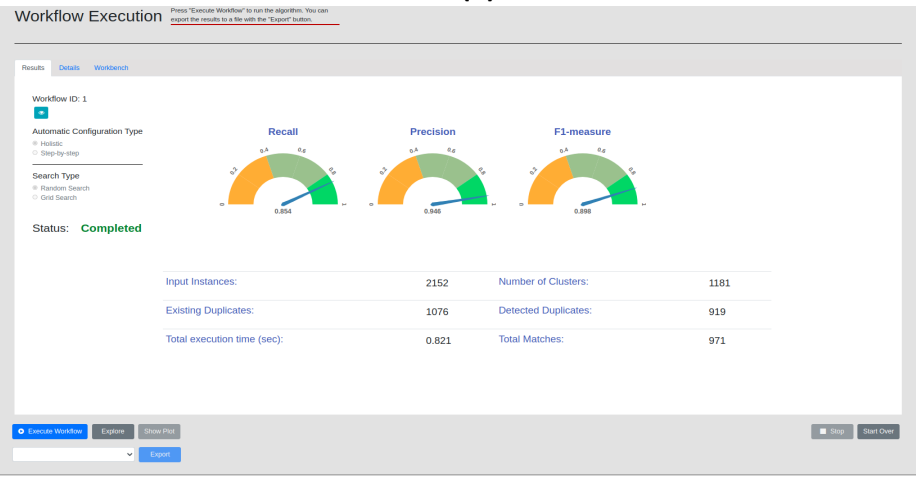

(f)

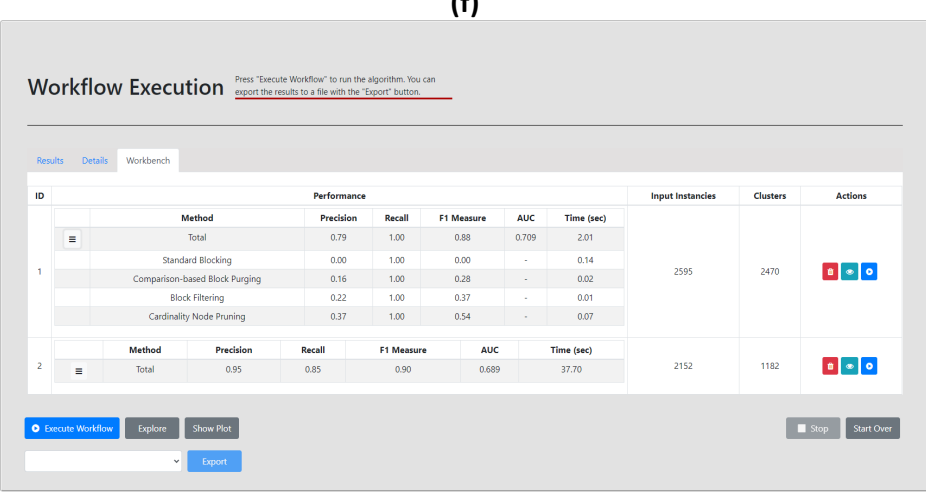

(h)

Figure 2: The screens of JedAl's Web application for reproducing all single core experiments in [1]: (a) The initial screen of JedAl's Web application. The button 'New Workflow' should be pressed. (b) The second screen, which defines the execution mode. The button 'Desktop Mode' should be pressed for the single-core experiments. (c) The third screen, which defines the type of the end-to-end pipeline. The button 'Run tests' should be pressed to start the reproduction of the experiments. (d) The fourth screen, which defines the experimental settings we want to reproduce with respect to the type of experiments, the type of ER, the type of end-to-end pipeline and the dataset. (e) The 'Confirm Configuration' screen that summarizes the experimental settings we have selected. (f) The final screen, 'Workflow Execution', which presents the performance of the selected end-to-end pipeline. (g) The screen showing the area under the curve of Progressive Recall (AUC) in case of Budget-awareness Tests. (h) The benchmark screen summarizing the performance of all pipelines executed so far with respect to Precision, Recall, F-Measure, Run-time and Progressive Recall (AUC), in case of Budget-awareness Tests. 
Table 8: The results of the Performance Tests over all real datasets across all testing platforms. For each pipeline, the effectiveness measures per dataset are common among all testing platforms. Only the running times differ among them. $I M$ indicates a test that was not carried out due to insufficient memory. Note that Precision, Recall and F-Measure are rounded to three decimal places, memory requirements to two decimal places and running times to one decimal place.

\begin{tabular}{|c|c|c|c|c|c|c|c|c|c|c|}
\hline & \multicolumn{8}{|c|}{ Clean-Clean ER } & \multicolumn{2}{|c|}{ Dirty ER } \\
\hline & $\begin{array}{c}\text { Restau- } \\
\text { rants } \\
\mathbf{D}_{\mathbf{c 1}}\end{array}$ & $\begin{array}{l}\text { Abt } \\
\text { Buy } \\
\text { D }_{\mathbf{c} 2}\end{array}$ & $\begin{array}{c}\text { Amazon } \\
\text { GP } \\
\mathbf{D}_{\mathbf{c 3}}\end{array}$ & $\begin{array}{c}\text { DBLP } \\
\text { ACM } \\
\mathbf{D}_{\mathbf{c} 4}\end{array}$ & $\begin{array}{c}\text { Walmart } \\
\text { Amazon } \\
\mathbf{D}_{\mathbf{c 5}}\end{array}$ & $\begin{array}{c}\text { DBLP } \\
\text { Scholar } \\
\text { D c6 }\end{array}$ & $\begin{array}{c}\text { IMDB } \\
\text { DBPedia } \\
\mathbf{D}_{\mathbf{c} 7}\end{array}$ & $\begin{array}{c}\text { DBP-3.0rc } \\
\text { DBP-3.4 } \\
\text { D }_{\mathbf{c 8}}\end{array}$ & $\mathbf{D}_{\text {cora }}$ & $\mathbf{D}_{\text {cddb }}$ \\
\hline Precision & 0.473 & 0.902 & 0.544 & 0.975 & 0.310 & 0.887 & 0.908 & 0.806 & 0.876 & 0.874 \\
\hline Recall & 1.000 & 0.836 & 0.653 & 0.988 & 0.878 & 0.952 & 0.834 & 0.819 & 0.816 & 0.856 \\
\hline F-Measure & 0.643 & 0.867 & 0.594 & 0.981 & 0.459 & 0.919 & 0.869 & 0.813 & 0.845 & 0.865 \\
\hline Memory (Gb) & 0.02 & 0.04 & 0.19 & 0.09 & 0.32 & 0.75 & 0.99 & 105.00 & 0.17 & 1.45 \\
\hline Ubuntu-base 1 & $1.1 \mathrm{sec}$ & $1.3 \mathrm{sec}$ & $12.0 \mathrm{sec}$ & $2.0 \mathrm{sec}$ & $8.3 \mathrm{sec}$ & $23.5 \mathrm{sec}$ & $91.0 \mathrm{sec}$ & $14.5 \mathrm{hrs}$ & $5.5 \mathrm{sec}$ & $65.0 \mathrm{sec}$ \\
\hline Ubuntu-base2 & $0.6 \mathrm{sec}$ & $1.3 \mathrm{sec}$ & $15.1 \mathrm{sec}$ & $1.3 \mathrm{sec}$ & $6.2 \mathrm{sec}$ & $28.9 \mathrm{sec}$ & $113.0 \mathrm{sec}$ & $22.1 \mathrm{hrs}$ & $2.7 \mathrm{sec}$ & $61.8 \mathrm{sec}$ \\
\hline Ubuntu - base3 & $0.5 \mathrm{sec}$ & $1.0 \mathrm{sec}$ & $11.2 \mathrm{sec}$ & $0.9 \mathrm{sec}$ & $4.4 \mathrm{sec}$ & $10.2 \mathrm{sec}$ & $68.0 \mathrm{sec}$ & $I M$ & $1.8 \mathrm{sec}$ & $30.6 \mathrm{sec}$ \\
\hline Ubuntu-base 4 & $0.2 \mathrm{sec}$ & $0.6 \mathrm{sec}$ & $8.6 \mathrm{sec}$ & $0.7 \mathrm{sec}$ & $3.5 \mathrm{sec}$ & $9.2 \mathrm{sec}$ & $53.4 \mathrm{sec}$ & $I M$ & $1.8 \mathrm{sec}$ & $23.4 \mathrm{sec}$ \\
\hline Ubuntu-base 5 & $0.3 \mathrm{sec}$ & $0.6 \mathrm{sec}$ & $8.2 \mathrm{sec}$ & $0.8 \mathrm{sec}$ & $3.7 \mathrm{sec}$ & $9.1 \mathrm{sec}$ & $48.5 \mathrm{sec}$ & $I M$ & $1.3 \mathrm{sec}$ & $23.9 \mathrm{sec}$ \\
\hline Ubuntu-base6 & $0.1 \mathrm{sec}$ & $0.6 \mathrm{sec}$ & $8.3 \mathrm{sec}$ & $0.7 \mathrm{sec}$ & $3.0 \mathrm{sec}$ & $7.7 \mathrm{sec}$ & $51.9 \mathrm{sec}$ & $I M$ & $1.3 \mathrm{sec}$ & $21.7 \mathrm{sec}$ \\
\hline Ubuntu-base 7 & $0.2 \mathrm{sec}$ & $1.3 \mathrm{sec}$ & $15.9 \mathrm{sec}$ & $1.2 \mathrm{sec}$ & $5.3 \mathrm{sec}$ & $15.3 \mathrm{sec}$ & $98.4 \mathrm{sec}$ & $16.8 \mathrm{hrs}$ & $2.4 \mathrm{sec}$ & $49.0 \mathrm{sec}$ \\
\hline Ubuntu-base8 & $0.2 \mathrm{sec}$ & $0.6 \mathrm{sec}$ & $7.9 \mathrm{sec}$ & $0.6 \mathrm{sec}$ & $2.5 \mathrm{sec}$ & $6.5 \mathrm{sec}$ & $39.5 \mathrm{sec}$ & $I M$ & $1.0 \mathrm{sec}$ & $18.8 \mathrm{sec}$ \\
\hline Ubuntu-base 9 & $0.4 \mathrm{sec}$ & $1.1 \mathrm{sec}$ & $16.1 \mathrm{sec}$ & $1.0 \mathrm{sec}$ & $5.1 \mathrm{sec}$ & $11.8 \mathrm{sec}$ & $75.5 \mathrm{sec}$ & $I M$ & $1.8 \mathrm{sec}$ & $30.8 \mathrm{sec}$ \\
\hline Windows - base 1 & $0.3 \mathrm{sec}$ & $1.0 \mathrm{sec}$ & $8.7 \mathrm{sec}$ & $0.9 \mathrm{sec}$ & $4.2 \mathrm{sec}$ & $18.2 \mathrm{sec}$ & $98.6 \mathrm{sec}$ & $I M$ & $1.7 \mathrm{sec}$ & $26.7 \mathrm{sec}$ \\
\hline
\end{tabular}

\begin{tabular}{|c|c|c|c|c|c|c|c|c|c|c|}
\hline \multicolumn{11}{|c|}{ (a) Default configuration of the budget- and schema-agnostic pipeline } \\
\hline Precision & 0.788 & 0.946 & 0.576 & 0.993 & 0.590 & 0.946 & 0.905 & 0.841 & 0.912 & 0.869 \\
\hline Recall & 1.000 & 0.854 & 0.646 & 0.992 & 0.753 & 0.949 & 0.876 & 0.821 & 0.819 & 0.886 \\
\hline F-Measure & 0.881 & 0.898 & 0.609 & 0.992 & 0.662 & 0.948 & 0.890 & 0.831 & 0.863 & 0.877 \\
\hline Memory (Gb) & 0.03 & 0.04 & 0.07 & 0.04 & 0.12 & 0.98 & 0.80 & 64.00 & 0.02 & 1.47 \\
\hline Ubuntu-base 1 & $1.0 \mathrm{sec}$ & $1.1 \mathrm{sec}$ & $4.5 \mathrm{sec}$ & $1.3 \mathrm{sec}$ & $5.3 \mathrm{sec}$ & $30.0 \mathrm{sec}$ & $46.0 \mathrm{sec}$ & $12.7 \mathrm{hrs}$ & $0.9 \mathrm{sec}$ & $65.7 \mathrm{sec}$ \\
\hline Ubuntu-base 2 & $0.7 \mathrm{sec}$ & $1.1 \mathrm{sec}$ & $6.1 \mathrm{sec}$ & $0.8 \mathrm{sec}$ & $12.9 \mathrm{sec}$ & $45.1 \mathrm{sec}$ & $49.5 \mathrm{sec}$ & $21.9 \mathrm{hrs}$ & $0.8 \mathrm{sec}$ & $70.0 \mathrm{sec}$ \\
\hline Ubuntu-base3 & $0.5 \mathrm{sec}$ & $0.8 \mathrm{sec}$ & $5.0 \mathrm{sec}$ & $0.6 \mathrm{sec}$ & $2.4 \mathrm{sec}$ & $16.4 \mathrm{sec}$ & $29.0 \mathrm{sec}$ & $I M$ & $0.6 \mathrm{sec}$ & $32.4 \mathrm{sec}$ \\
\hline Ubuntu-base 4 & $0.5 \mathrm{sec}$ & $0.7 \mathrm{sec}$ & $4.1 \mathrm{sec}$ & $0.4 \mathrm{sec}$ & $1.8 \mathrm{sec}$ & $12.6 \mathrm{sec}$ & $23.9 \mathrm{sec}$ & $I M$ & $0.4 \mathrm{sec}$ & $25.6 \mathrm{sec}$ \\
\hline Ubuntu-base 5 & $0.4 \mathrm{sec}$ & $0.6 \mathrm{sec}$ & $3.2 \mathrm{sec}$ & $0.7 \mathrm{sec}$ & $2.0 \mathrm{sec}$ & $12.2 \mathrm{sec}$ & $24.5 \mathrm{sec}$ & $I M$ & $0.5 \mathrm{sec}$ & $30.8 \mathrm{sec}$ \\
\hline Ubuntu-base6 & $0.1 \mathrm{sec}$ & $0.5 \mathrm{sec}$ & $3.1 \mathrm{sec}$ & $0.5 \mathrm{sec}$ & $1.7 \mathrm{sec}$ & $13.1 \mathrm{sec}$ & $21.8 \mathrm{sec}$ & $I M$ & $0.3 \mathrm{sec}$ & $23.3 \mathrm{sec}$ \\
\hline Ubuntu-base7 & $0.1 \mathrm{sec}$ & $0.6 \mathrm{sec}$ & $7.3 \mathrm{sec}$ & $0.8 \mathrm{sec}$ & $2.8 \mathrm{sec}$ & $23.9 \mathrm{sec}$ & $41.7 \mathrm{sec}$ & $16.5 \mathrm{hrs}$ & $0.7 \mathrm{sec}$ & $51.3 \mathrm{sec}$ \\
\hline Ubuntu-base8 & $0.1 \mathrm{sec}$ & $0.4 \mathrm{sec}$ & $3.5 \mathrm{sec}$ & $0.4 \mathrm{sec}$ & $1.2 \mathrm{sec}$ & $11.2 \mathrm{sec}$ & $18.6 \mathrm{sec}$ & $12.9 \mathrm{hrs}$ & $0.3 \mathrm{sec}$ & $24.5 \mathrm{sec}$ \\
\hline Ubuntu-base 9 & $0.2 \mathrm{sec}$ & $0.4 \mathrm{sec}$ & $5.9 \mathrm{sec}$ & $0.6 \mathrm{sec}$ & $2.2 \mathrm{sec}$ & $16.8 \mathrm{sec}$ & $34.8 \mathrm{sec}$ & $I M$ & $0.3 \mathrm{sec}$ & $34.9 \mathrm{sec}$ \\
\hline Windows - base 1 & $0.2 \mathrm{sec}$ & $0.6 \mathrm{sec}$ & $4.8 \mathrm{sec}$ & $0.5 \mathrm{sec}$ & $2.3 \mathrm{sec}$ & $18.7 \mathrm{sec}$ & $28.9 \mathrm{sec}$ & $I M$ & $0.5 \mathrm{sec}$ & $32.5 \mathrm{sec}$ \\
\hline \multicolumn{11}{|c|}{ (b) Best configuration of the budget- and schema-agnostic pipeline } \\
\hline Precision & 0.755 & 0.884 & 0.663 & 0.978 & 0.829 & 0.953 & 0.931 & 0.833 & 0.751 & 0.278 \\
\hline Recall & 0.933 & 0.438 & 0.423 & 0.932 & 0.552 & 0.775 & 0.499 & 0.370 & 0.859 & 0.719 \\
\hline F-Measure & 0.834 & 0.585 & 0.517 & 0.954 & 0.663 & 0.855 & 0.649 & 0.512 & 0.802 & 0.401 \\
\hline Memory (Gb) & 0.01 & 0.02 & 0.02 & 0.02 & 0.06 & 0.11 & 0.42 & 30.00 & 0.02 & 0.06 \\
\hline Ubuntu-base 1 & $0.2 \mathrm{sec}$ & $0.4 \mathrm{sec}$ & $0.5 \mathrm{sec}$ & $0.6 \mathrm{sec}$ & $0.5 \mathrm{sec}$ & $14.0 \mathrm{sec}$ & $7.7 \mathrm{sec}$ & $15.2 \mathrm{~min}$ & $0.3 \mathrm{sec}$ & $0.6 \mathrm{sec}$ \\
\hline Ubuntu-base 2 & $0.2 \mathrm{sec}$ & $0.2 \mathrm{sec}$ & $0.2 \mathrm{sec}$ & $0.5 \mathrm{sec}$ & $0.2 \mathrm{sec}$ & $13.8 \mathrm{sec}$ & $6.9 \mathrm{sec}$ & $12.4 \mathrm{~min}$ & $0.3 \mathrm{sec}$ & $0.3 \mathrm{sec}$ \\
\hline Ubuntu-base3 & $0.2 \mathrm{sec}$ & $0.3 \mathrm{sec}$ & $0.3 \mathrm{sec}$ & $0.2 \mathrm{sec}$ & $0.2 \mathrm{sec}$ & $10.6 \mathrm{sec}$ & $5.2 \mathrm{sec}$ & $I M$ & $0.2 \mathrm{sec}$ & $0.3 \mathrm{sec}$ \\
\hline Ubuntu-base 4 & $0.1 \mathrm{sec}$ & $0.1 \mathrm{sec}$ & $0.1 \mathrm{sec}$ & $0.1 \mathrm{sec}$ & $0.1 \mathrm{sec}$ & $10.2 \mathrm{sec}$ & $3.5 \mathrm{sec}$ & $I M$ & $0.2 \mathrm{sec}$ & $0.3 \mathrm{sec}$ \\
\hline Ubuntu-base 5 & $0.1 \mathrm{sec}$ & $0.1 \mathrm{sec}$ & $0.2 \mathrm{sec}$ & $0.2 \mathrm{sec}$ & $0.3 \mathrm{sec}$ & $7.4 \mathrm{sec}$ & $3.4 \mathrm{sec}$ & $I M$ & $0.2 \mathrm{sec}$ & $0.3 \mathrm{sec}$ \\
\hline Ubuntu-base6 & $0.1 \mathrm{sec}$ & $0.1 \mathrm{sec}$ & $0.2 \mathrm{sec}$ & $0.3 \mathrm{sec}$ & $0.1 \mathrm{sec}$ & $6.3 \mathrm{sec}$ & $3.3 \mathrm{sec}$ & $I M$ & $0.1 \mathrm{sec}$ & $0.3 \mathrm{sec}$ \\
\hline Ubuntu-base7 & $0.1 \mathrm{sec}$ & $0.2 \mathrm{sec}$ & $0.2 \mathrm{sec}$ & $0.2 \mathrm{sec}$ & $0.2 \mathrm{sec}$ & $14.2 \mathrm{sec}$ & $7.7 \mathrm{sec}$ & $11.0 \mathrm{~min}$ & $0.1 \mathrm{sec}$ & $0.2 \mathrm{sec}$ \\
\hline Ubuntu-base8 & $0.1 \mathrm{sec}$ & $0.1 \mathrm{sec}$ & $0.1 \mathrm{sec}$ & $0.1 \mathrm{sec}$ & $0.1 \mathrm{sec}$ & $5.9 \mathrm{sec}$ & $3.2 \mathrm{sec}$ & $5.2 \mathrm{~min}$ & $0.1 \mathrm{sec}$ & $0.1 \mathrm{sec}$ \\
\hline Ubuntu-base 9 & $0.1 \mathrm{sec}$ & $0.1 \mathrm{sec}$ & $0.2 \mathrm{sec}$ & $0.2 \mathrm{sec}$ & $0.1 \mathrm{sec}$ & $16.5 \mathrm{sec}$ & $5.6 \mathrm{sec}$ & $I M$ & $0.1 \mathrm{sec}$ & $0.2 \mathrm{sec}$ \\
\hline Windows - base 1 & $0.1 \mathrm{sec}$ & $0.1 \mathrm{sec}$ & $0.2 \mathrm{sec}$ & $0.2 \mathrm{sec}$ & $0.1 \mathrm{sec}$ & $16.5 \mathrm{sec}$ & $5.6 \mathrm{sec}$ & $I M$ & $0.1 \mathrm{sec}$ & $0.2 \mathrm{sec}$ \\
\hline
\end{tabular}

(c) Best configuration of the budget-agnostic, schema-based pipeline

The outcomes of the Performance, the Scalability and the ${ }_{355}$ Budget-awareness tests over all testing platforms are reported ${ }_{356}$ in Tables 8, 9 and 10, respectively. In all cases, the effec-357 tiveness measures are common among all platforms, with the 358 only differences corresponding to the running times. Compared 359 to the experiments reported in [1], the effectiveness results of 360 Budget-awareness tests are practically identical in most cases.361
The only significant exceptions pertain to the best schemaagnostic pipeline over $D_{c 2}, D_{c 3}$ and $D_{c d d b}$, whose F-Measure has now changed from $0.900,0.607$ and 0.872 to $0.898,0.609$ and 0.877 , respectively, after some bug fixes. The F-Measure of the default schema-agnostic pipeline over $D_{c 3}$ has also increased from 0.586 to 0.594 . The effectiveness results of the Scalability and the Budget-awareness tests are also identical 
Table 9: The results of the Scalability Tests over the seven synthetic datasets across all testing platforms. For each pipeline, the effectiveness measures per dataset are common among all testing platforms. Only the running times differ among them. IM indicates a test that was not carried out due to insufficient memory. Note that Precision, Recall and F-Measure are rounded to three decimal places, memory requirements to two decimal places and running times to one decimal place.

\begin{tabular}{|c|c|c|c|c|c|c|c|}
\hline & $\mathrm{D}_{10 \mathrm{~K}}$ & $\mathbf{D}_{50 \mathrm{~K}}$ & $\mathrm{D}_{100 \mathrm{~K}}$ & $\mathbf{D}_{200 K}$ & $\mathbf{D}_{300 K}$ & $\mathbf{D}_{1 \mathrm{M}}$ & $\mathbf{D}_{2 M}$ \\
\hline Precision & 0.948 & 0.899 & 0.887 & 0.844 & 0.866 & 0.868 & 0.836 \\
\hline Recall & 0.994 & 0.989 & 0.983 & 0.978 & 0.973 & 0.960 & 0.954 \\
\hline F-Measure & 0.970 & 0.942 & 0.933 & 0.906 & 0.916 & 0.911 & 0.891 \\
\hline Memory (Gb) & 0.12 & 0.80 & 3.10 & 6.20 & 7.20 & 15.00 & 25.00 \\
\hline Ubuntu-base 1 & $1.8 \mathrm{sec}$ & $12.8 \mathrm{sec}$ & $35.1 \mathrm{sec}$ & $120.2 \mathrm{sec}$ & $193.1 \mathrm{sec}$ & $32.3 \mathrm{~min}$ & $147.1 \mathrm{~min}$ \\
\hline Ubuntu-base 2 & $1.6 \mathrm{sec}$ & $11.4 \mathrm{sec}$ & $37.3 \mathrm{sec}$ & $130.8 \mathrm{sec}$ & $199.3 \mathrm{sec}$ & $33.4 \mathrm{~min}$ & $145.4 \mathrm{~min}$ \\
\hline Ubuntu-base3 & $1.4 \mathrm{sec}$ & $5.2 \mathrm{sec}$ & $19.8 \mathrm{sec}$ & $51.9 \mathrm{sec}$ & $141.9 \mathrm{sec}$ & $22.1 \mathrm{~min}$ & $I M$ \\
\hline Ubuntu-base 4 & $0.9 \mathrm{sec}$ & $4.3 \mathrm{sec}$ & $10.7 \mathrm{sec}$ & $54.3 \mathrm{sec}$ & $I M$ & $I M$ & $I M$ \\
\hline Ubuntu-base 5 & $1.0 \mathrm{sec}$ & $3.7 \mathrm{sec}$ & $11.5 \mathrm{sec}$ & $37.8 \mathrm{sec}$ & $77.0 \mathrm{sec}$ & $16.9 \mathrm{~min}$ & $I M$ \\
\hline Ubuntu-base6 & $0.7 \mathrm{sec}$ & $4.2 \mathrm{sec}$ & $10.8 \mathrm{sec}$ & $36.7 \mathrm{sec}$ & $114.6 \mathrm{sec}$ & - & - \\
\hline Ubuntu-base 7 & $0.8 \mathrm{sec}$ & $6.3 \mathrm{sec}$ & $19.9 \mathrm{sec}$ & $63.4 \mathrm{sec}$ & $148.0 \mathrm{sec}$ & $24.2 \mathrm{~min}$ & $93.5 \mathrm{~min}$ \\
\hline Ubuntu-base8 & $0.5 \mathrm{sec}$ & $3.6 \mathrm{sec}$ & $9.0 \mathrm{sec}$ & $27.1 \mathrm{sec}$ & $71.3 \mathrm{sec}$ & $12.9 \mathrm{~min}$ & $51.4 \mathrm{~min}$ \\
\hline Ubuntu - base9 & $1.5 \mathrm{sec}$ & $8.2 \mathrm{sec}$ & $15.7 \mathrm{sec}$ & $46.9 \mathrm{sec}$ & $118.4 \mathrm{sec}$ & $22.6 \mathrm{~min}$ & $I M$ \\
\hline Windows - base 1 & $1.4 \mathrm{sec}$ & $5.0 \mathrm{sec}$ & $10.8 \mathrm{sec}$ & $47.2 \mathrm{sec}$ & $232.5 \mathrm{sec}$ & $I M$ & $I M$ \\
\hline
\end{tabular}

(a) Default configuration of the budget- and schema-agnostic pipeline

\begin{tabular}{|l|r|r|r|r|r|r|r|}
\hline Precision & 1.000 & 1.000 & 1.000 & 1.000 & 1.000 & 1.000 & 1.000 \\
Recall & 0.593 & 0.598 & 0.602 & 0.600 & 0.602 & 0.603 & 0.602 \\
F-Measure & 0.744 & 0.749 & 0.752 & 0.750 & 0.751 & 0.752 & 0.752 \\
\hline Memory (Gb) & 0.03 & 0.10 & 0.30 & 1.15 & 1.75 & 11.00 & 16.00 \\
\hline Ubuntu - base1 & $7.0 \mathrm{sec}$ & $137.2 \mathrm{sec}$ & $695.3 \mathrm{sec}$ & $55.6 \mathrm{~min}$ & $140.3 \mathrm{~min}$ & $17.8 \mathrm{hrs}$ & $>40 \mathrm{hrs}$ \\
Ubuntu - base 2 & $5.3 \mathrm{sec}$ & $120.3 \mathrm{sec}$ & $534.8 \mathrm{sec}$ & $49.6 \mathrm{~min}$ & $96.9 \mathrm{~min}$ & $19.3 \mathrm{hrs}$ & $>40 \mathrm{hrs}$ \\
Ubuntu - base3 & $4.0 \mathrm{sec}$ & $89.4 \mathrm{sec}$ & $367.8 \mathrm{sec}$ & $26.3 \mathrm{~min}$ & $69.4 \mathrm{~min}$ & $13.0 \mathrm{hrs}$ & $>40 \mathrm{hrs}$ \\
Ubuntu - base4 & $3.9 \mathrm{sec}$ & $74.6 \mathrm{sec}$ & $316.5 \mathrm{sec}$ & $24.0 \mathrm{~min}$ & $48.4 \mathrm{~min}$ & $I M$ & $I M$ \\
Ubuntu - base5 & $3.6 \mathrm{sec}$ & $67.9 \mathrm{sec}$ & $298.2 \mathrm{sec}$ & $21.3 \mathrm{~min}$ & $55.9 \mathrm{~min}$ & $10.3 \mathrm{hrs}$ & $>40 \mathrm{hrs}$ \\
Ubuntu - base6 & $3.8 \mathrm{sec}$ & $78.6 \mathrm{sec}$ & $341.5 \mathrm{sec}$ & $23.6 \mathrm{~min}$ & $57.1 \mathrm{~min}$ & - & $>40 \mathrm{hrs}$ \\
Ubuntu - base7 & $7.9 \mathrm{sec}$ & $172.2 \mathrm{sec}$ & $704.1 \mathrm{sec}$ & $49.5 \mathrm{~min}$ & $111.9 \mathrm{~min}$ & $19.8 \mathrm{hrs}$ & $>40 \mathrm{hrs}$ \\
Ubuntu - base8 & $3.1 \mathrm{sec}$ & $140.1 \mathrm{sec}$ & $375.2 \mathrm{sec}$ & $22.3 \mathrm{~min}$ & $49.7 \mathrm{~min}$ & $10.2 \mathrm{hrs}$ & $39.8 \mathrm{hrs}$ \\
Ubuntu - base9 & $5.3 \mathrm{sec}$ & $96.8 \mathrm{sec}$ & $376.4 \mathrm{sec}$ & $28.7 \mathrm{~min}$ & $64.5 \mathrm{~min}$ & $10.8 \mathrm{hrs}$ & $>40 \mathrm{hrs}$ \\
\hline Windows - base 1 & $4.3 \mathrm{sec}$ & $87.3 \mathrm{sec}$ & $376.7 \mathrm{sec}$ & $26.7 \mathrm{~min}$ & $56.8 \mathrm{~min}$ & $I M$ & $I M$ \\
\hline
\end{tabular}

(b) Best configuration of the budget-agnostic, schema-based pipeline

with those reported in [1]; only their format has changed from ${ }_{379}$ diagrams to tables. In all cases, the running times in [1] are reproduced here, corresponding to Ubuntu - base 1 in Tables $8^{80}$ and 9 and to Ubuntu - base $1^{\prime}$ in Table 10

Finally, it is worth stressing that there is a delay when pressing the 'Next' button in the window 'Entity Matching' of ${ }^{386}$ the schema-agnostic pipelines. For small datasets, the delay is ${ }^{387}$ hardly observable, but it increases for larger datasets, raising up up $_{38}$ to few minutes for $D_{1 M}, D_{2 M}$ and $D_{c 8}$. This delay is caused by 389 a process that converts all entity profiles into the representation ${ }_{390}$ model of the selected Entity Matching method. This is included 391 in the running times of Ubuntu - base1, where all experiments 392 were run through script files, but is not considered by any other ${ }_{393}$ testing platform, where all experiments were executed through ${ }_{394}$ JedAl's user interface. This is one of the reasons for the signif-395 icantly higher running times of Ubuntu - base 1 even in com-396 parison to similar testing platforms, like Ubuntu-base $2 . \quad 397$

\section{Reconfiguring and Extending our Experiments}

\subsection{Evaluating different experimental setups}

To test the robustness of our experimental study, the configuration of a particular experiment can be adjusted in two different ways as follows:

1. by enriching or modifying the methods of at least one pipeline step, and/or

2. by altering the value of at least one parameter in one of the selected methods.

This is possible by repeating the procedure in Table 7 up to the first window of Step 10, namely 'Data Reading'. Subsequently, in the separate window of each step, the pre-selected options can be modified as described below, in Sections 3.1.1. 3.1.2 and 3.1.3 for each type of experiments.

Note that every method in every pipeline step is associated with three configuration approaches: 'Default', 'Automatic', 'Manual'. The 'Default' configuration is already widely used in the experimental analysis of [1]. The 'Automatic' configuration applies grid or random search over numerous iterations 
Table 10: The results of the Budget-awareness Tests over all real datasets across all testing platforms. For each pipeline, effectiveness is measured through the area under the curve of Progressive Recall, which is common among all testing platforms in each dataset only for the budget-aware pipeline. Its budget-agnostic counterpart arranges all pairwise comparisons in a random order, thus yielding a Progressive Recall that differs in each run and, thus, among the testing platforms. Note that Precision, Recall and F-Measure are rounded to three decimal places, memory requirements to two decimal places and running times to one decimal place. Note also that $D_{c 8}$ is omitted, as in [1], due to the excessively large running time and the very high memory requirements of the corresponding experiment.

\begin{tabular}{|c|c|c|c|c|c|c|c|c|c|}
\hline & \multicolumn{7}{|c|}{ Clean-Clean ER } & \multicolumn{2}{|c|}{ Dirty ER } \\
\hline & $\begin{array}{c}\text { Restau- } \\
\text { rants } \\
\mathbf{D}_{\mathbf{c 1}}\end{array}$ & $\begin{array}{l}\text { Abt } \\
\text { Buy } \\
\text { D } 22\end{array}$ & $\begin{array}{c}\text { Amazon } \\
\text { GP } \\
\mathbf{D}_{\mathbf{c 3}}\end{array}$ & $\begin{array}{c}\text { DBLP } \\
\text { ACM } \\
\mathbf{D}_{\mathbf{c 4}}\end{array}$ & $\begin{array}{c}\text { Walmart } \\
\text { Amazon } \\
\mathbf{D}_{\mathbf{c 5}}\end{array}$ & $\begin{array}{c}\text { DBLP } \\
\text { Scholar } \\
\text { Dc6 }\end{array}$ & $\begin{array}{c}\text { IMDB } \\
\text { DBPedia } \\
\mathbf{D}_{\mathbf{c} 7}\end{array}$ & $\mathbf{D}_{\text {cora }}$ & $\mathbf{D}_{\text {cddb }}$ \\
\hline Progressive Recall & 0.709 & 0.689 & 0.573 & 0.866 & 0.635 & 0.930 & 0.616 & 0.416 & 0.585 \\
\hline Memory (Gb) & 0.06 & 0.08 & 0.30 & 0.16 & 0.65 & 4.00 & 6.00 & 0.30 & 3.50 \\
\hline Ubuntu-base $1^{\prime}$ & $0.5 \mathrm{sec}$ & $13.7 \mathrm{sec}$ & $1.8 \mathrm{~min}$ & $32.9 \mathrm{sec}$ & $5.2 \mathrm{~min}$ & $46.3 \mathrm{~min}$ & $18.4 \mathrm{hrs}$ & $16.9 \mathrm{sec}$ & $79.3 \mathrm{sec}$ \\
\hline Ubuntu - base2 & $0.6 \mathrm{sec}$ & $19.0 \mathrm{sec}$ & $3.4 \mathrm{~min}$ & $49.8 \mathrm{sec}$ & $7.8 \mathrm{~min}$ & $68.2 \mathrm{~min}$ & $20.1 \mathrm{hrs}$ & $15.8 \mathrm{sec}$ & $96.5 \mathrm{sec}$ \\
\hline Ubuntu-base3 & $0.4 \mathrm{sec}$ & $14.6 \mathrm{sec}$ & $2.2 \mathrm{~min}$ & $48.5 \mathrm{sec}$ & $7.4 \mathrm{~min}$ & $54.1 \mathrm{~min}$ & $12.7 \mathrm{hrs}$ & $12.2 \mathrm{sec}$ & $73.5 \mathrm{sec}$ \\
\hline Ubuntu-base 4 & $0.3 \mathrm{sec}$ & $12.5 \mathrm{sec}$ & $1.5 \mathrm{~min}$ & $35.7 \mathrm{sec}$ & $6.1 \mathrm{~min}$ & $40.7 \mathrm{~min}$ & $I M$ & $9.9 \mathrm{sec}$ & $55.6 \mathrm{sec}$ \\
\hline Ubuntu-base 5 & $0.6 \mathrm{sec}$ & $12.2 \mathrm{sec}$ & $2.0 \mathrm{~min}$ & $31.5 \mathrm{sec}$ & $5.1 \mathrm{~min}$ & $37.2 \mathrm{~min}$ & $10.8 \mathrm{hrs}$ & $9.4 \mathrm{sec}$ & $49.9 \mathrm{sec}$ \\
\hline Ubuntu-base6 & $0.3 \mathrm{sec}$ & $11.9 \mathrm{sec}$ & $1.4 \mathrm{~min}$ & $33.5 \mathrm{sec}$ & $4.9 \mathrm{~min}$ & $34.5 \mathrm{~min}$ & $9.6 \mathrm{hrs}$ & $10.1 \mathrm{sec}$ & $53.8 \mathrm{sec}$ \\
\hline Ubuntu-base 7 & $0.4 \mathrm{sec}$ & $20.9 \mathrm{sec}$ & $2.7 \mathrm{~min}$ & $63.1 \mathrm{sec}$ & $9.4 \mathrm{~min}$ & $64.7 \mathrm{~min}$ & $22.0 \mathrm{hrs}$ & $19.6 \mathrm{sec}$ & $107.8 \mathrm{sec}$ \\
\hline Ubuntu-base 8 & $0.3 \mathrm{sec}$ & $10.2 \mathrm{sec}$ & $1.2 \mathrm{~min}$ & $30.0 \mathrm{sec}$ & $4.2 \mathrm{~min}$ & $28.7 \mathrm{~min}$ & $9.5 \mathrm{hrs}$ & $8.8 \mathrm{sec}$ & $45.8 \mathrm{sec}$ \\
\hline Ubuntu-base9 & $0.8 \mathrm{sec}$ & $16.6 \mathrm{sec}$ & $2.2 \mathrm{~min}$ & $48.8 \mathrm{sec}$ & $6.9 \mathrm{~min}$ & $38.8 \mathrm{~min}$ & $13.9 \mathrm{hrs}$ & $14.3 \mathrm{sec}$ & $66.9 \mathrm{sec}$ \\
\hline Windows - base 1 & $0.5 \mathrm{sec}$ & $14.9 \mathrm{sec}$ & $1.9 \mathrm{~min}$ & $20.4 \mathrm{sec}$ & $8.3 \mathrm{~min}$ & $52.4 \mathrm{~min}$ & $11.3 \mathrm{hrs}$ & $15.3 \mathrm{sec}$ & $80.0 \mathrm{sec}$ \\
\hline
\end{tabular}

(a) Budget-aware, schema-agnostic pipeline

\begin{tabular}{|c|c|c|c|c|c|c|c|c|c|}
\hline Memory (Gb) & 0.09 & 0.20 & 0.20 & 0.35 & 0.65 & 4.00 & 6.00 & 0.30 & 3.50 \\
\hline Progressive Recall & 0.489 & 0.418 & 0.337 & 0.491 & 0.386 & 0.478 & 0.435 & 0.661 & 0.451 \\
\hline Ubuntu - base $1^{\prime}$ & $1.6 \mathrm{sec}$ & $19.2 \mathrm{sec}$ & $2.4 \mathrm{~min}$ & $34.4 \mathrm{sec}$ & $11.6 \mathrm{~min}$ & $51.0 \mathrm{~min}$ & $20.8 \mathrm{hrs}$ & $30.4 \mathrm{sec}$ & $13.5 \mathrm{~min}$ \\
\hline Progressive Recall & 0.491 & 0.400 & 0.341 & 0.489 & 0.383 & 0.479 & 0.436 & 0.665 & 0.446 \\
\hline Ubuntu-base2 & $0.5 \mathrm{sec}$ & $15.5 \mathrm{sec}$ & $2.6 \mathrm{~min}$ & $40.4 \mathrm{sec}$ & $13.6 \mathrm{~min}$ & $61.5 \mathrm{~min}$ & $21.8 \mathrm{hrs}$ & $18.5 \mathrm{sec}$ & $13.6 \mathrm{~min}$ \\
\hline Progressive Recall & 0.481 & 0.403 & 0.328 & 0.488 & 0.397 & 0.474 & 0.437 & 0.659 & 0.466 \\
\hline Ubuntu-base3 & $0.4 \mathrm{sec}$ & $12.4 \mathrm{sec}$ & $1.9 \mathrm{~min}$ & $31.4 \mathrm{sec}$ & $12.6 \mathrm{~min}$ & $49.5 \mathrm{~min}$ & $14.5 \mathrm{hrs}$ & $15.4 \mathrm{sec}$ & $11.9 \mathrm{~min}$ \\
\hline Progressive Recall & 0.521 & 0.405 & 0.335 & 0.495 & 0.371 & 0.488 & $I M$ & 0.668 & 0.464 \\
\hline Ubuntu - base 4 & $0.5 \mathrm{sec}$ & $10.2 \mathrm{sec}$ & $1.6 \mathrm{~min}$ & $25.9 \mathrm{sec}$ & $9.7 \mathrm{~min}$ & $36.7 \mathrm{~min}$ & $I M$ & $10.4 \mathrm{sec}$ & $7.8 \mathrm{~min}$ \\
\hline Progressive Recall & 0.487 & 0.402 & 0.322 & 0.488 & 0.383 & 0.475 & 0.435 & 0.678 & 0.482 \\
\hline Ubuntu-base 5 & $0.5 \mathrm{sec}$ & $11.1 \mathrm{sec}$ & $1.4 \mathrm{~min}$ & $25.8 \mathrm{sec}$ & $8.8 \mathrm{~min}$ & $37.3 \mathrm{~min}$ & $12.2 \mathrm{hrs}$ & $10.8 \mathrm{sec}$ & $8.0 \mathrm{~min}$ \\
\hline Progressive Recall & 0.483 & 0.399 & 0.326 & 0.498 & 0.374 & 0.476 & 0.436 & 0.666 & 0.460 \\
\hline Ubuntu - base6 & $0.2 \mathrm{sec}$ & $10.7 \mathrm{sec}$ & $1.6 \mathrm{~min}$ & $28.9 \mathrm{sec}$ & $9.0 \mathrm{~min}$ & $35.6 \mathrm{~min}$ & $11.1 \mathrm{hrs}$ & $10.6 \mathrm{sec}$ & $8.0 \mathrm{~min}$ \\
\hline Progressive Recall & 0.457 & 0.406 & 0.344 & 0.501 & 0.381 & 0.463 & 0.436 & 0.661 & 0.510 \\
\hline Ubuntu - base 7 & $0.4 \mathrm{sec}$ & $21.9 \mathrm{sec}$ & $2.9 \mathrm{~min}$ & $57.4 \mathrm{sec}$ & $19.0 \mathrm{~min}$ & $72.7 \mathrm{~min}$ & $23.3 \mathrm{hrs}$ & $21.8 \mathrm{sec}$ & $15.9 \mathrm{~min}$ \\
\hline Progressive Recall & 0.528 & 0.416 & 0.319 & 0.502 & 0.376 & 0.464 & 0.435 & 0.668 & 0.488 \\
\hline Ubuntu-base8 & $0.2 \mathrm{sec}$ & $9.0 \mathrm{sec}$ & $1.4 \mathrm{~min}$ & $25.1 \mathrm{sec}$ & $7.5 \mathrm{~min}$ & $29.8 \mathrm{~min}$ & $10.8 \mathrm{hrs}$ & $9.9 \mathrm{sec}$ & $6.9 \mathrm{~min}$ \\
\hline Progressive Recall & 0.453 & 0.414 & 0.330 & 0.490 & 0.379 & 0.476 & 0.436 & 0.669 & 0.463 \\
\hline Ubuntu-base 9 & $0.3 \mathrm{sec}$ & $14.4 \mathrm{sec}$ & $2.4 \mathrm{~min}$ & $39.7 \mathrm{sec}$ & $12.4 \mathrm{~min}$ & $46.4 \mathrm{~min}$ & $14.5 \mathrm{hrs}$ & $18.0 \mathrm{sec}$ & $11.9 \mathrm{~min}$ \\
\hline Progressive Recall & 0.520 & 0.396 & 0.334 & 0.498 & 0.381 & 0.469 & 0.434 & 0.659 & 0.453 \\
\hline Windows - base 1 & $0.7 \mathrm{sec}$ & $14.5 \mathrm{sec}$ & $2.2 \mathrm{~min}$ & $37.9 \mathrm{sec}$ & $13.8 \mathrm{~min}$ & $50.8 \mathrm{~min}$ & $13.5 \mathrm{hrs}$ & $15.1 \mathrm{sec}$ & $24.3 \mathrm{~min}$ \\
\hline
\end{tabular}

(b) Budget- and schema-agnostic pipeline 


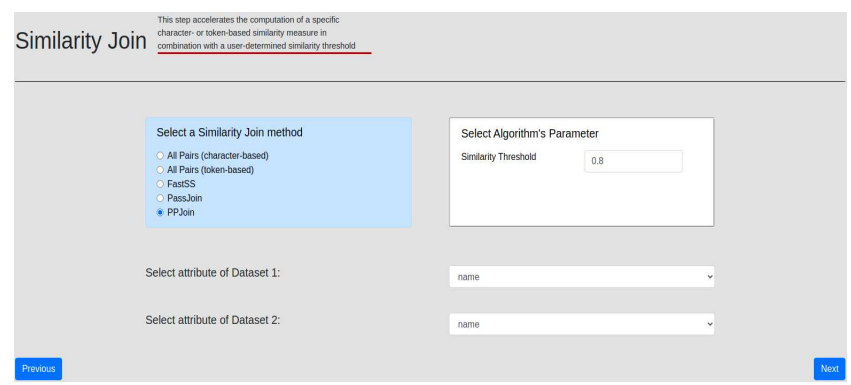

(a)

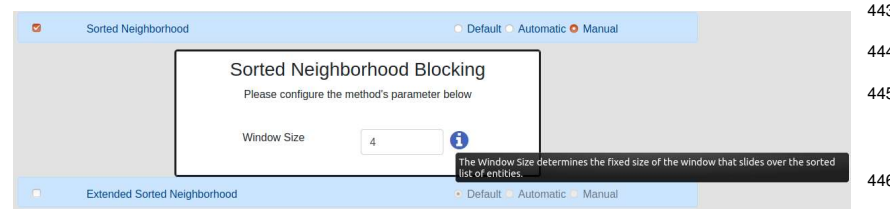

(b)

Figure 3: (a) The screen showing the configuration for a particular pipeline step. (b) The tooltip that explains the role of a particular parameter during the ${ }^{448}$ manual configuration of a method.

so as to identify the settings that maximize F-Measure. The ${ }^{451}$ random search involves 100 iterations, while the grid search $^{452}$ might yield an exponential number of iterations in case multi-453 ple parameters are simultaneously fine-tuned. As both options ${ }^{454}$ might lead to long running times, the preferred approach is the ${ }^{455}$ 'Manual' configuration. After selecting it, JedAI presents all parameters of the current pipeline along with their default val-456 ues, as in Figure 3(a). The user can alter these values at will and ${ }_{457}$ store them by pressing 'Next' to proceed to the next window. ${ }_{458}$

Note also that every method in JedAl implements the ${ }_{459}$ IDocumentation interface, which conveys all necessary in- 460 formation for its manual configuration. When configuring $\mathrm{a}_{461}$ specific parameter, the information image $(i)$ is shown. When 462 leaving the mouse cursor over it, a tooltip appears that describes 463 the role of this parameter. An example is shown in Figure 3 b).464

Below, we explain the restrictions that apply to each pipeline 465 step with respect to the methods that can be selected.

\subsubsection{Schema-Agnostic End-to-End Pipeline}

As explained above, this pipeline involves six steps:

1. Schema Clustering. At most one method can be selected, 470 but this step is not used in the considered experiments. 471

2. Block Building. One or more of the nine available methods can be selected. All experiments exclusively employ 472 Token Blocking, which is a parameter-free approach.

3. Block Cleaning. Any combination of the three available 474 methods is possible. All experiments apply Comparison- ${ }_{475}$ based Block Purging and Block Filtering with their default ${ }_{476}$ parameter values.

\section{7}

4. Comparison Cleaning. At most one of the nine available ${ }_{478}$ methods can be selected. In our experiments, we exclu- 479 sively use Cardinality Node Pruning (CNP) with its de- -480 fault configuration. All methods are configured simply by ${ }_{481}$ selecting one of the six weighting schemes.

482

5. Entity Matching. One of the two available methods can ${ }_{483}$ be applied. All experiments employ the Profile Matcher.484
Both methods are configured by selecting a similarity measure and a compatible representation model, which transforms the set of textual attribute values in each entity profile into a suitable format. These two parameters give rise to numerous configurations.

6. Entity Clustering. At most one method can be selected in this step. There are three methods available for CleanClean ER, but all experiments employ the Unique Mapping Clustering approach. For Dirty ER, there are seven methods for Dirty ER, but all experiments use the Connected Components Clustering. All methods are configured by setting their similarity threshold, below which all pairwise comparisons are discarded.

\subsubsection{Schema-Based End-to-End Pipeline}

This pipeline consists of two steps:

1. The Similarity Join step offers five similarity join algorithms. Among them, PPJoin is used in all experiments. All methods are configured by setting their similarity threshold along with the attribute(s), to which they are applied.

2. The Entity Clustering step is the same as the schemaagnostic pipeline. In most cases, it uses the same similarity threshold as the previous step.

\subsubsection{Budget-Aware Schema-Agnostic Pipeline}

This pipeline differs from its budget-agnostic counterpart (see Section 3.1.1 only in the Prioritization step that intervenes between Comparison Cleaning and Entity Matching. There are different options for this step, depending on the preceding pipeline steps: if no Block Building method is employed, two methods are available, otherwise one of five different methods can be used. The latter approach was used in all Budgetawareness tests. In both cases, at most one approach can be selected and it is configured by setting its budget (i.e., number of executed comparisons) and the weighting scheme that lies at its core.

Note that for all tests, the next configuration experiment is performed by pressing the 'Start Over' button at the bottom right corner of Figure 2 (f) to return to the Data Reading step of the current experiment.

\subsection{Extending our experiments}

Our experimental study can be extended in two ways. First, by adding new datasets through the 'Data Reading' step. The window of this step allows users to select any dataset in any of the supported formats (CSV, relational DB, XML or RDF) that is stored either locally or is available through a server with a public URL. Note that each dataset should be accompanied by the golden standard comprising all duplicates.

Second, it is possible to extent our experimental analysis with new methods in any of the considered pipeline steps by leveraging JedAl's extensible architecture. The only requirement is that every new method is available through a Java class that implements the interface of the corresponding pipeline step - as 
explained in [1], every step is associated with a simple Java in-532 terface that determines its input and output. In this way, new methods can be seamlessly integrated into JedAl's code and533 be treated like the already available methods. Ideally, the new534 methods should also implement the IDocumentation inter-535 face, which exposes the following functions that return textual536 descriptions about the core characteristics of an algorithm: $\quad{ }^{537}$

- getMethodName() returns the name of the method.

- getParameterName(int parameterId) returns the ${ }_{54}^{540}$ name of a particular configuration parameter.

- getParameterDescription(int parameterId) returns a short description for a particular configuration ${ }^{544}$ parameter.

- getMethodParameters () returns a description for all ${ }^{547}$ configuration parameters of the method, using the above ${ }_{548}^{548}$ functions.

- getMethodInfo() returns a short description of the $e^{551}$ method's internal functionality.

- getMethodConfiguration() returns the parameter con- ${ }^{554}$ figuration of the current instance of a method. It is called ${ }^{555}$ by logger.

- getParameterConfiguration() returns a JsonArray 558 object with a JsonObject for every configuration pa-559 rameter that comprises the following information: the ${ }^{560}$ class of the parameter (e.g., java.lang. Integer), its ${ }^{561}$ name, determined by the function getParameterName,562 its default, minimum and maximum values along with the ${ }^{563}$ step one, and its description, determined by the function ${ }^{564}$ getParameterDescription. This information is used565 for the manual configuration through JedAI's interface. $\quad 566$

This documentation, which is also leveraged by JedAl's user interface, ensures that new methods can be easily employed by ${ }_{569}$ users other than their creators. For more details on extending ${ }_{570}$ JedAl please refer to [1].

\section{Conclusions}

We have presented an analytical user guide for JedAl's Web 576 application, which is available through a Docker image. Our 577 instructions allow a user with limited or no familiarity with En-578 tity Resolution to repeat all single-core experiments in [1] s0579 as to evaluate the relative performance of the main end-to-end 580 pipelines. Our instructions also facilitate the reconfiguration of ${ }_{581}$ these experiments, by constructing and evaluating pipelines of ${ }_{582}$ arbitrary complexity.

All these experiments involve learning-free methods. In the 584 future, we plan to extend JedAl with learning-based methods,585 paying particular attention to the integration of Deep Learning 586 technologies.

\section{Revision Comments}

This reproducibility manuscript is a valuable complement to the parent paper [1], where the last release of JedAl software was presented. JedAl includes a web-based user interface and a complete library of techniques needed to create end-to-end Entity Resolution (ER) pipelines. The authors compared different ER techniques by considering three different dimensions that included: (a) Schema-awareness, (b) Budget-awareness, and (c) Execution mode. The wide set of experiments provided included the evaluation of 17 datasets and considered the performance, scalability, and budget awareness of the ER pipelines. This paper provides the actual configuration used for those ER pipelines, and gives some ideas regarding how they can be personalized. Furthermore, some guidelines showing how JedAl can be extended are also devised.

Apart from creating a permanent repository in Mendeley with the necessary software and datasets, the authors provide a Docker-based system to reproduce those experiments. Using the web-based interface of JedAl, any researcher can easily use the default configuration parameters provided for each experiment, execute it, and finally see the results of that execution. Besides, JedAl also allows to configure and personalize those default parameters, as well as the addition of new methods for the comparison with existing methods, adding extra value to the current work.

While reviewing this manuscript, a few issues around reproducibility were brought into the discussion, which show how difficult it can be to provide a complete reproducible framework. We dealt with some experiments where the provided default parameters were wrong, which led to unexpected results. Another minor issue was related to yielding slightly different values than those reported in the parent paper or figures showing the results in a rather different shape. We also found some mismatches concerning the memory requirements needed to run some experiments, which would not end or report higher execution times than expected. All those issues were successfully fixed during the revision process. The authors satisfactorily took all our comments into account and improved their software library and web application. Finally, the JedAI reproduction framework does not provide a mechanism to automatically run all the experiments, gather all the results, and create the same tables and figures of the parent paper, which would be extremely interesting to reproduce the original work easily. However, the workflow included in JedAl still allows any researcher to effortlessly reproduce each experiment. The process consists of choosing the experiment to perform, going through the screens that display the default parameters, starting the execution, waiting for it to complete, and finally gathering the results.

We would like to thank the authors for their considerable effort to provide a valuable software library to the research community. This library allows new researchers to understand and reproduce state-of-the-art experiments with minimal effort and guarantees long-term software support, following a sequence of precise and straightforward instructions.

Acknowledgements. This work was partially funded by the 


\section{References}

[1] G. Papadakis, G. Mandilaras, L. Gagliardelli, G. Simonini,652 E. Thanos, G. Giannakopoulos, S. Bergamaschi, T. Palpanas, 653 M. Koubarakis, Three-dimensional entity resolution with jedai, 654 Inf. Syst. 93 (2020) 101565.

[2] G. Papadakis, E. Ioannou, E. Thanos, T. Palpanas, The four gen-656 erations of entity resolution, Synthesis Lectures on Data Man-657 agement.

[3] P. Christen, Data Matching - Concepts and Techniques for659 Record Linkage, Entity Resolution, and Duplicate Detection,660 Data-Centric Systems and Applications, Springer, 2012.

[4] X. L. Dong, D. Srivastava, Big Data Integration, Synthesis Lec-662 tures on Data Management, Morgan \& Claypool Publishers,663 2015.

[5] V. Christophides, V. Efthymiou, K. Stefanidis, Entity Resolution665 in the Web of Data, Synthesis Lectures on the Semantic Web:666 Theory and Technology, Morgan \& Claypool Publishers, 2015. 667

[6] A. K. Elmagarmid, P. G. Ipeirotis, V. S. Verykios, Duplicate668 record detection: A survey, IEEE Trans. Knowl. Data Eng. 19 (1)669 (2007) 1-16.

[7] G. Papadakis, D. Skoutas, E. Thanos, T. Palpanas, Blocking and filtering techniques for entity resolution: A survey, ACM Computing Surveys 53 (2) (2020) 31:1-31:42.

[8] V. Christophides, V. Efthymiou, T. Palpanas, G. Papadakis, K. Stefanidis, An overview of end-to-end entity resolution for big data, ACM Computing Surveys 53 (6).

[9] L. Getoor, A. Machanavajjhala, Entity resolution: Theory, practice \& open challenges, PVLDB 5 (12) (2012) 2018-2019.

[10] K. Stefanidis, V. Efthymiou, M. Herschel, V. Christophides, Entity resolution in the web of data, in: WWW, 2014, pp. 203-204.

[11] G. Papadakis, T. Palpanas, Web-scale, schema-agnostic, end-toend entity resolution, in: The Web Conference (WWW), Lyon, France, 2018.

[12] G. Papadakis, E. Ioannou, T. Palpanas, Entity resolution: Past, present and yet-to-come, in: EDBT, 2020, pp. 647-650.

[13] G. Papadakis, Entity resolution benchmark dataset, https:// data.mendeley.com/datasets/4whpm32y47 (2020).

[14] J. Euzenat, A. Ferrara, C. Meilicke, J. Pane, F. Scharffe, P. Shvaiko, H. Stuckenschmidt, O. Sváb-Zamazal, V. Svátek, C. T. dos Santos, Results of the ontology alignment evaluation initiative 2010, in: Proceedings of the 5th International Workshop on Ontology Matching (OM-2010), 2010.

[15] Ontology alignment evaluation initiative, http://oaei. ontologymatching. org/2010 (2010).

[16] H. Köpcke, A. Thor, E. Rahm, Evaluation of entity resolution approaches on real-world match problems, Proc. VLDB Endow. 3 (1) (2010) 484-493.

[17] Benchmark datasets for entity resolution, https: //dbs.uni-leipzig.de/research/projects/object_ matching/benchmark_datasets_for_entity_resolution (2010).

[18] C. Gokhale, S. Das, A. Doan, J. F. Naughton, N. Rampalli, J. W. Shavlik, X. Zhu, Corleone: hands-off crowdsourcing for entity matching, in: SIGMOD, 2014, pp. 601-612.

[19] S. Das, A. Doan, P. S. G. C., C. Gokhale, P. Konda, Y. Govind, D. Paulsen, The magellan data repository, https://sites. google.com/site/anhaidgroup/projects/data

[20] G. Papadakis, E. Ioannou, C. Niederée, P. Fankhauser, Efficient entity resolution for large heterogeneous information spaces, in: WSDM, 2011, pp. 535-544.

[21] G. Papadakis, Blocking framework, https://sourceforge. net/projects/erframework/

[22] A. McCallum, K. Nigam, L. H. Ungar, Efficient clustering of high-dimensional data sets with application to reference matching, in: ACM SIGKDD, 2000, pp. 169-178.

[23] Repeatability datasets, https://hpi.de/naumann/ projects/repeatability/datasets.html.

[24] U. Draisbach, F. Naumann, A comparison and generalization of blocking and windowing algorithms for duplicate detection, in: Proceedings of the International Workshop on Quality in Databases (QDB), 2009, pp. 51-56.

[25] B. Kenig, A. Gal, Mfiblocks: An effective blocking algorithm for entity resolution, Inf. Syst. 38 (6) (2013) 908-926.

[26] P. Konda, S. Das, P. S. G. C., A. Doan, A. Ardalan, J. R. Ballard, H. Li, F. Panahi, H. Zhang, J. F. Naughton, S. Prasad, G. Krishnan, R. Deep, V. Raghavendra, Magellan: Toward building entity matching management systems, Proc. VLDB Endow. 9 (12) (2016) 1197-1208.

[27] S. Mudgal, H. Li, T. Rekatsinas, A. Doan, Y. Park, G. Krishnan, R. Deep, E. Arcaute, V. Raghavendra, Deep learning for entity matching: A design space exploration, in: SIGMOD, 2018, pp. 19-34. 